\title{
Nonautonomous Semilinear Parabolic Equations on an Infinite Interval
}

\author{
Jason R. Morris \\ Communicated by Y. Charles Li, received February 24, 2006 \\ and, in revised form, May 20, 2006.
}

\begin{abstract}
The topological degree for Fredholm operators is used to prove the existence of solutions to a class of semilinear parabolic partial differential equations on an infinite time interval. General initial conditions are allowed and homogeneous Dirichlet boundary conditions are assumed. The solutions are shown not only to exist for all times, but also to be $L^{p}$ in time and space.
\end{abstract}

\section{CONTEnTs}

1. Introduction

2. The functional setting

3. The Fredholm property

4. Properness on closed bounded subsets 218

5. Existence of solutions by degree theory 222

6. Application to semilinear parabolic problems 224

$\begin{array}{ll}\text { References } & 232\end{array}$

2000 Mathematics Subject Classification. Primary 35K55; Secondary 35B40, 35B45, 47H11.

Key words and phrases. Topological degree, Fredholm operator, Dirichlet boundary condition, semilinear parabolic partial differential equation.

This work is an elaboration of part of the author's doctoral dissertation [22], completed at the University of Pittsburgh under the supervision of Professor Patrick J. Rabier. The author gratefully thanks Professor Rabier for his guidance and support. 


\section{Introduction}

In this paper we consider the existence of strong solutions to semilinear parabolic partial differential equations of the following form:

$$
\begin{aligned}
u_{t}-A(t) u+F(t, x, u) & =f(t, x) \text { in }[0, \infty) \times \Omega, \\
u(0, \cdot) & =g(x) \text { in } L^{p}(\Omega), \\
u(t, x) & =0 \text { on }[0, \infty) \times \partial \Omega .
\end{aligned}
$$

In the above, $\Omega$ is a bounded domain in $\mathbb{R}^{d}$ and $A=A(t)$ is a path of bounded linear operators on (say) $W^{2, p}(\Omega)$. Our purpose is to find conditions on $A$ and on the nonlinearity $F$ such that the above problem is solvable for all reasonable $L^{p}$ right sides $(f, g)$. We seek solutions that not only exist for all times, but that are also of $L^{p}$ type in time and space. This is a much different problem than mere local existence, or even existence on a fixed but bounded time interval $[0, T]$.

1.1. Background. There is a vast literature in the general area of semilinear parabolic partial differential equations, and much of the literature is devoted to local existence of solutions to various problems. A survey of those papers devoted to global existence reveals that quite a lot of work has been put into the semilinear heat equation $u_{t}-\Delta u=|u|^{\gamma}$. The usual line of inquiry is whether solutions exist for all times or blow up in finite time; this study goes back to Fujita [12]. The study of this kind of problem continues with various modifications in the nonlinearity, various choices of dimension and domain, and so on. See for example Baras and Kersner [3], Gu and Sun [13], Kwembe [16], Maatoug and Riahi [20], Marinov and Georgiev [21], Pinsky [25], Poláčik and Yanagida [26], and Weissler [36] and the references therein. The results in this paper differ from that program in at least the following respects. We are interested primarily in a nonautonomous operator $A(t)$, although $A(t)=\Delta$ is certainly allowed. Also, we obtain solutions with a specific asymptotic property (beyond membership in $L^{p}$ ). In addition, we wish to allow a variety of right hand sides $f \in L^{p}$. Finally, we will find general conditions on the nonlinearity, rather than work in specifics.

The results given in Pazy [23, Chapter 8] are more illustrative of the problem at hand. In particular, we refer to Theorem 8.2.7, Theorem 8.3.5, and Theorem 8.4.4. The first of these results incorporates the asymptotic behavior of the solutions, but for a specific homogeneous problem $\left(d=1, A(t)=\frac{\partial^{2}}{\partial x^{2}}\right.$, and $F=F(u)$ is Hölder continuous). The second result is also for a specific problem, and gives only local existence. The third result is rather general, but again gives only local existence.

Some papers that prove existence of solutions in a more general setting are as follows. For an autonomous equation, existence is proved for sufficiently small right sides by Racke [32]. Other various general results may be found in Constantin, Escher, and Yin [5, section 3], Carvalho and Cholewa [4], and Kirane and Tatar $[\mathbf{1 5}]$. The hypotheses and conclusions vary, yet none of these results include any information about the behavior of solutions as $t \rightarrow \infty$.

Very recently, a general treatment of quasilinear parabolic problems was given by Amann [2]. Part of that paper is devoted to the question of existence in a setting strictly more general than that of the present paper; we do not consider quasilinear problems that are not semilinear. Amann gives a sufficient condition for a solution to be continuable to the entire interval in which the problem is posed. However, all of this is done in a fixed and bounded interval $[0, T]$. Presumably, one can then let 
$T \rightarrow \infty$ to possibly obtain a solution on $[0, \infty)$. Once again, one would lose any information on the behavior of $u$ as $t \rightarrow \infty$.

We refer the reader to Henry [14], Lieberman [17], and Lunardi [19] for further elements of the theory of parabolic equations. Note that in $[\mathbf{1 7}]$ the emphasis is on autonomous equations in bounded time-space domains (which need not be cylinders). In [19], which employs Hölder spaces rather than Sobolev spaces, existence is proved on a bounded interval using a priori estimates for local solutions. On an unbounded interval, the asymptotic stability of stationary solutions is obtained for autonomous problems with small initial data (and also for periodic solutions of periodic problems). Our results for nonautonomous problems impose no such smallness condition on the initial data, and our use of the common Hölder condition on $A$ is limited to finding a priori bounds in our example (Lemma 6.10) but is not needed in principle (Theorem 5.3).

1.2. Overview of method and results. The present paper illustrates one way to use what is known in the linear setting to obtain existence results for related nonlinear problems. We rely on the literature to handle the linear part of the problem, which we will generally assume to satisfy an isomorphism property. For the linear theory, we refer to Rabier $[\mathbf{2 7}, \mathbf{2 8}]$ and Schnaubelt $[\mathbf{3 3}, \mathbf{3 4}]$ and references therein. We then use a degree theoretic procedure to find conditions on the nonlinearity that preserve the existence of solutions.

Because of the absence of required compactness properties (in the form of a Sobolev embedding), the degree theory that we use is that of Pejsachowicz and Rabier [24] (and also [11] with Fitzpatrick), rather than the usual Leray-Schauder degree. When proving existence by a homotopy argument, the newer degree theory is used exactly like the old, except that the Fredholm and properness properties of the functional operator must be checked. Note that these properties would have been consequences of the very compactness that is missing. Most of this paper is devoted to finding practical conditions on $A$ and $F$ to ensure that the various abstract requirements of the degree argument are met.

The degree argument is composed of several phases. After deciding on an appropriate functional setting (anisotropic Sobolev spaces), we recast the differential equation as a single equation $\Phi(u)=(f, g)$. To use the degree for Fredholm maps, we have to arrange that $\Phi$ is $C^{1}$. We show that this is the case under a uniform continuity requirement for $A$, and a mild smoothness condition for $F$. In particular, we require that $F$ be $C^{1}$, but only in the third argument, and we do not require any Hölder continuity in $A$ or the first two arguments of $F$. Section 2 is devoted to these considerations.

We then show in Section 3 that at every point $u$, the linearization $D \Phi(u)$ is a compact perturbation of $D \Phi(0)$. Thus, the Fredholm property and index needed for the degree argument will rely only on the linearization at $u=0$.

A more substantial obstacle is the required properness of $\Phi$ on the closed bounded subsets of the domain space. We will demonstrate the equivalence of this property with the simpler condition that certain auxiliary equations of the form $u_{t}-B(t) u+G(t, x, u)=0$ have no nonzero solutions in the anisotropic Sobolev space on the whole line (even though the original problem is posed on the half line). This is the subject of Section 4. 
In Section 5 we use a degree argument to prove Theorem 5.3, our main result. We emphasize that because of the choice of domain space for $\Phi$, solutions $u$ guaranteed by Theorem 5.3 not only exist for all times, but have well known asymptotic properties. In particular, $u$ is $L^{p}$ jointly in time and space and $u(t, x)$ converges to 0 uniformly in $x$ as $t \rightarrow \infty$.

To prove Theorem 5.3, we use the results up to that point to replace the abstract requirements of the degree argument with concrete conditions on the PDE. The only requirement that is left untouched is that one should have a priori bounds for solutions of the PDE as the right sides are deformed to zero. The techniques for a priori bounds vary from problem to problem. One example is provided by the application presented in Section 6. In Section 6, we use Theorem 5.3 to prove the existence of a solution under several more explicit conditions. The bulk of the work in Section 6 is devoted to a priori bounds. We first find bounds in $L^{p}$ using a variant of the Poincaré inequality and an energy method. We then find bounds in $L^{\infty}$, by analyzing the result of evaluating $u_{t}-A(t) u+F(t, x, u)$ along a sequence of maximizers of mollifications of a solution $u$. Assuming that the linearized problem is an isomorphism, this is enough to obtain the desired bounds.

Properties of Bochner integration are used throughout the paper. All needed background can be found in Diestel and Uhl [7], Dunford and Schwartz [8], Edwards [9], and succinctly in the final appendix in Evans [10]. Background on degree theory may be found in Deimling [6] and Lloyd [18]. As we have already mentioned, we use the degree theory of $[\mathbf{2 4}, \mathbf{1 1}]$, but in practice the arguments are nearly the same.

\section{The functional setting}

2.1. Properties of the function spaces. We begin by identifying several Banach spaces of functions. Let $\Omega$ be a bounded domain in $\mathbb{R}^{d}$. We do not need to place any explicit conditions on $\partial \Omega$. However, $\partial \Omega$ will need some smoothness in order to verify the first condition in our main result, Theorem 5.3. See Remark 5.4 for details.

Fix once and for all a positive real number $p>d+1$. Let $^{1}$

$$
X:=L^{p}(\Omega),
$$

and

$$
W:=W^{2, p}(\Omega) \cap W_{0}^{1, p}(\Omega) .
$$

Then incorporate the time variable via

$$
\mathcal{X}:=L^{p}((0, \infty), X)
$$

which is isometrically isomorphic to $L^{p}((0, \infty) \times \Omega)$ in the canonical way. In light of this isometry, we will use expressions such as $u(t), u(t, \cdot)$, and $u(t)(\cdot)$ interchangeably. Next, we define the subspace $\mathcal{W}$ of $\mathcal{X}$ by

$$
\mathcal{W}:=L^{p}((0, \infty), W) \cap W^{1, p}((0, \infty), X) .
$$

The space $\mathcal{W}$ is isometrically isomorphic to the subspace of the Banach space $L^{p}((0, \infty) \times \Omega)$ consisting of functions that

(1) have distributional derivatives that are in $L^{p}$, up to second order in the $\Omega$ variables,

\footnotetext{
${ }^{1}$ We use LHS $:=$ RHS to emphasize that the equality is a definition of LHS.
} 
(2) have first order distributional derivatives in $L^{p}$ in the $(0, \infty)$ variable,

(3) and vanish on $(0, \infty) \times \partial \Omega$.

If one were to remove the third condition, the resulting space is often denoted by $W_{p}^{1,2}((0, \infty) \times \Omega)$, endowed with the expected norm:

$$
\|u\|_{1,2}:=\|u\|_{p}+\left\|u_{t}\right\|_{p}+\sum_{|\alpha| \leq 2}\left\|D_{x}^{\alpha} u\right\|_{p}
$$

where $\|\cdot\|_{p}$ is the norm in $L^{p}((0, \infty) \times \Omega)$. Recall that for an open domain $U$ in $\mathbb{R}^{d+1}$ and an exponent $0<\lambda \leq 1$, the notation $C^{0, \lambda}(\bar{U})$ denotes the Banach space of all bounded, uniformly continuous functions on $U$ that satisfy a Hölder estimate of exponent $\lambda\left(|u(y)-u(z)| \leq M|y-z|^{\lambda}\right)$. See Adams and Fournier [1, Chapter 1] or Evans [10, Chapter 5] for details. According to the Sobolev Embedding Theorem for $W^{1, p}((0, \infty) \times \Omega)$ (also in $[\mathbf{1}]$ ), the space $\mathcal{W}$ embeds continuously in $C^{0, \lambda}(\overline{(0, \infty) \times \Omega})$ for each $0<\lambda \leq 1-(d+1) / p$.

By this Hölder continuity, the functions in $\mathcal{W}$ are bounded and uniformly continuous on $[0, \infty) \times \bar{\Omega}$. Indeed, each bounded subset of $\mathcal{W}$ is uniformly bounded and uniformly equicontinuous on $[0, \infty) \times \bar{\Omega}$. We prove more in Lemma 2.2 , which is used in the proof of Theorem 3.2.

REMARK 2.1. This embedding into a Hölder space is why we insist that $p$ be greater than $d+1$. Note that the boundary of $\Omega$ need not be regular for this purpose, because the functions in $\mathcal{W}$ vanish when $x \in \partial \Omega$ and can be boundedly extended past the bottom of the cylinder to vanish on the boundary of a larger cylinder.

Lemma 2.2. Let $\mathcal{H}$ be a bounded subset of $\mathcal{W}$. There are constants $C_{1}, C_{2}>0$ and $0<\mu_{1}, \mu_{2}<1$ such that for all $u \in \mathcal{H}$,

$$
\|u\|_{L^{\infty}((0, \infty) \times \Omega)} \leq C_{1}\|u\|_{L^{p}((0, \infty) \times \Omega)}^{\mu_{1}},
$$

Proof. We sketch the proof of (2.2). The proof of (2.1) is similar. Since $\mathcal{H}$ is bounded, there is a Hölder estimate

$$
\left|u(t, x)-u\left(t^{\prime}, x^{\prime}\right)\right| \leq M\left(\left|t-t^{\prime}\right|+\left|x-x^{\prime}\right|\right)^{\lambda},
$$

where $M>0$ depends on $\mathcal{H}$ (and where we are using the continuous representative for $u$ ). Let $t \geq 0$, and let $x \in \Omega$ be such that $|u(t, x)|$ is a maximum over $\Omega$; recall that $u(t, \cdot)$ vanishes on $\partial \Omega$ for all $t \geq 0$. Taking $\delta:=(|u(t, x)| / 2 M)^{1 / \lambda}$, we find that

$$
\left|u\left(t, x^{\prime}\right)\right| \geq \frac{1}{2}|u(t, x)|
$$

as long as $\left|x-x^{\prime}\right|<\delta$. Integration of $p^{\text {th }}$ powers of both sides of (2.4) with respect to $x^{\prime}$ will demonstrate that

$$
\begin{aligned}
\|u(t, \cdot)\|_{L^{p}(\Omega)}^{p} & \geq\|u(t, \cdot)\|_{L^{p}\left(\left\{x^{\prime}|| x-x^{\prime} \mid<\delta\right\}\right)}^{p} \\
& \geq \omega_{d} \delta^{d} \frac{1}{2^{p}}|u(t, x)|^{p} \\
& =C|u(t, x)|^{(d+\lambda p) / \lambda},
\end{aligned}
$$


where $\omega_{d}$ is the volume of the unit ball in $\mathbb{R}^{d}$. This inequality is equivalent to $(2.2)$, and so completes the proof.

We note that results such as the above lemma are often obtained by interpolation, but the above proof seemed simple enough to include.

We will also make use of the space $\mathcal{W}(0)$ of traces of functions in $\mathcal{W}$ :

$$
\mathcal{W}(0):=\{u(0) \mid u \in \mathcal{W}\} \subset X .
$$

This vector space is well defined by virtue of the uniform continuity of functions in $W^{1, p}((0, \infty), X)$ and is a Banach space with norm induced by the quotient of $\mathcal{W}$ by the kernel of the map $u \mapsto u(0)$. That is, $\|v\|_{\mathcal{W}(0)}:=\inf \left\{\|u\|_{\mathcal{W}} \mid u(0)=v\right\}$. We set $\mathcal{Y}:=\mathcal{X} \times \mathcal{W}(0)$.

2.2. The smoothness of the Nemytskii operator. We will now define an operator $\Phi: \mathcal{W} \rightarrow \mathcal{Y}$. Let a path of operators $A=A(t)$,

$$
A:[0, \infty) \rightarrow \mathcal{L}\left(W^{2, p}(\Omega), L^{p}(\Omega)\right)
$$

be given. (We use $\mathcal{L}(W, X)$ for the space of all bounded linear operators from $W$ into $X$.) It is assumed throughout this paper that

(H-A1) $\quad A$ is bounded and uniformly continuous on $[0, \infty)$.

We assume that $F=F(t, x, z):[0, \infty) \times \Omega \times \mathbb{R} \rightarrow \mathbb{R}$ is such that

$$
D_{z} F \text { exists everywhere, }
$$

and for each bounded open subinterval $J$ of $\mathbb{R}$,

$$
F \text { and } D_{z} F \text { are in } C^{0}(\overline{(0, \infty) \times \Omega \times J}) \text {. }
$$

That is, $F$ and $D_{z} F$ are bounded and uniformly continuous on the set $(0, \infty) \times \Omega \times J$. Finally,

$$
F(t, x, 0)=D_{z} F(t, x, 0)=0 \quad \forall(t, x) \in[0, \infty) \times \Omega .
$$

The above hypotheses concerning $A$ and $F$ will henceforth be collectively named "(H)".

To $A$ we associate the Nemytskii operator $\mathbf{A}$ on $\mathcal{W}$; for each $u \in \mathcal{W}$, the function $\mathbf{A} u:[0, \infty) \rightarrow X$ is defined as follows:

$$
(\mathbf{A} u)(t):=A(t) u(t) .
$$

Similarly, we associate to $F$ an operator $\mathbf{F}$ on $\mathcal{W}$. For each $u \in \mathcal{W}$, a function $\mathbf{F}(u):[0, \infty) \times \Omega \rightarrow \mathbb{R}$ is defined by

$$
(\mathbf{F}(u))(t, x):=F(t, x, u(t, x)) .
$$

We can now define $\Phi: \mathcal{W} \rightarrow \mathcal{Y}$ via

$$
\Phi(u):=\left(\frac{\mathrm{d} u}{\mathrm{~d} t}-\mathbf{A} u+\mathbf{F}(u), u(0)\right)
$$

for each $u \in \mathcal{W}$. That $\Phi$ is well defined by (2.5) is part of the conclusion of the following lemma. 
LEMMA 2.3. With the four hypotheses $(\mathrm{H})$, the operator $\Phi$ defined in (2.5) is a well defined and $C^{1}$ operator from $\mathcal{W}$ into $\mathcal{Y}$. The derivative is given by

$$
D \Phi(u) v=\left(\frac{\mathrm{d} v}{\mathrm{~d} t}-\mathbf{A} v+\mathbf{G}(u) v, v(0)\right),
$$

where $G:=D_{z} F$ and the multiplication $\mathbf{G}(u) v$ is pointwise.

Proof. We discuss each of four addends that comprise (2.5). First, the operator $\frac{\mathrm{d}}{\mathrm{d} t}$ is linear and continuous from $W^{1, p}((0, \infty), X)$ into $\mathcal{X}$ by definition of $W^{1, p}$. Second, the evaluation map $u \mapsto u(0) \in \mathcal{W}(0)$ is linear and continuous by definition of the norm in $\mathcal{W}(0)$.

Third, by hypothesis (H-A1), let $M<\infty$ be a bound for the operator norm $\|A(t)\|$ of $A(t)$ as $t$ ranges over $[0, \infty)$. Thus, $\|\mathbf{A} u\|_{\mathcal{X}}^{p} \leq \int_{0}^{\infty} M^{p}\|u(t)\|_{W}^{p} \mathrm{~d} t \leq$ $M^{p}\|u\|_{\mathcal{W}}^{p}$, so that $\mathbf{A}$ is continuous from $\mathcal{W}$ into $\mathcal{X}$. Therefore, the three linear terms are continuous from $\mathcal{W}$ into $\mathcal{Y}$.

To show that $\mathbf{F}$ maps $\mathcal{W}$ into $\mathcal{X}$, fix $u \in \mathcal{W}$. By hypothesis (H-F2), let $M<\infty$ be a bound for $\left|D_{z} F\right|$ on $(0, \infty) \times \Omega \times$ rge $u$. (Recall that $u$ is bounded on $[0, \infty) \times \Omega$.) Since

$$
\begin{aligned}
\mathbf{F}(u)(t, x) & =\int_{0}^{1} \frac{\mathrm{d}}{\mathrm{d} s} F(t, x, s u(t, x)) \mathrm{d} s \\
& =u(t, x) \int_{0}^{1} D_{z} F(t, x, s u(t, x)) \mathrm{d} s,
\end{aligned}
$$

it follows that $|\mathbf{F}(u)(t, x)| \leq M|u(t, x)|$, which shows that $\mathbf{F}(u)$ is in $\mathcal{X}$. We now prove that $\mathbf{F}$ is differentiable.

Fix $u \in \mathcal{W}$ and let $G:=D_{z} F$. By hypothesis $(\mathrm{H}-\mathrm{F} 2), \mathbf{G}(u) \in L^{\infty}((0, \infty) \times \Omega)$. Therefore, pointwise multiplication by $\mathbf{G}(u)$ is a bounded linear map from $\mathcal{W}$ into $\mathcal{X}$ (even from $\mathcal{X}$ into $\mathcal{X})$. We need to show that

$$
\frac{\mathbf{F}(u+v)-\mathbf{F}(u)-\mathbf{G}(u) v}{\|v\|_{\mathcal{W}}} \rightarrow 0 \text { in } \mathcal{X} \text { as } v \rightarrow 0 \text { in } \mathcal{W} .
$$

Since $F(t, x, u(t, x)+v(t, x))-F(t, x, u(t, x))-D_{z} F(t, x, u(t, x)) v(t, x)$ can instead be written as

$$
\int_{0}^{1} \frac{\mathrm{d}}{\mathrm{d} s} F(t, x, u(t, x)+s v(t, x)) \mathrm{d} s-D_{z} F(t, x, u(t, x)) v(t, x),
$$

which is just

$$
v(t, x) \int_{0}^{1}\left\{D_{z} F(t, x, u(t, x)+s v(t, x))-D_{z} F(t, x, u(t, x))\right\} \mathrm{d} s,
$$

we find that

$$
\begin{aligned}
(\mathbf{F}(u+v)-\mathbf{F}(u)-\mathbf{G}(u) & v)(t, x) \\
& =v \int_{0}^{1}\left\{D_{z} F(t, x, u+s v)-D_{z} F(t, x, u)\right\} \mathrm{d} s,
\end{aligned}
$$

where we have suppressed the dependence of $u$ and $v$ on $(t, x)$.

By the Sobolev embedding of $\mathcal{W}$ into a Hölder space (or just into $C^{0}$ ), there is $\delta>0$ such that $|v(t, x)|<1$ whenever $\|v\|_{\mathcal{W}}<\delta$. Because of hypothesis (H-F2), 
the function $D_{z} F$ is uniformly continuous on $(0, \infty) \times \Omega \times(u([0, \infty) \times \bar{\Omega})+[-1,1])$. Thus, given $\epsilon>0$, there is $\delta_{1}>0$ such that

$$
\left|D_{z} F(t, x, u(t, x)+s v(t, x))-D_{z} F(t, x, u(t, x))\right|<\epsilon
$$

for all $(s, t, x) \in[0,1] \times[0, \infty) \times \Omega$, provided that $|v(t, x)|<\min \left\{1, \delta_{1}\right\}$ for all $(t, x)$. By further reduction of $\delta$, we ensure that this is the case. By (2.8) and (2.9),

$$
|(\mathbf{F}(u+v)-\mathbf{F}(u)-\mathbf{G}(u) v)(t, x)| \leq \epsilon|v(t, x)|
$$

whence

$$
\|\mathbf{F}(u+v)-\mathbf{F}(u)-\mathbf{G}(u) v\|_{\mathcal{X}} \leq \epsilon\|v\|_{\mathcal{X}}
$$

whenever $\|v\|_{\mathcal{W}}<\delta$. Since $\|v\|_{\mathcal{X}} \leq\|v\|_{\mathcal{W}}$, this verifies (2.7).

It remains to verify that $\mathbf{G}$ is a continuous map of $\mathcal{W}$ into $\mathcal{L}(\mathcal{W}, \mathcal{X})$. Given $u \in \mathcal{W}$ and $\epsilon>0$, let $\delta$ be chosen as in the preceding paragraph, so that, by $(2.9)$ with $s=1$,

$$
\left|D_{z} F(t, x, u(t, x)+v(t, x))-D_{z} F(t, x, u(t, x))\right|<\epsilon
$$

whenever $\|v\|_{\mathcal{W}}<\delta$. It follows that for such $v$ and for any $w \in \mathcal{W}$,

$$
|[(\mathbf{G}(u+v)-\mathbf{G}(u)) w](t, x)| \leq \epsilon|w(t, x)|
$$

so that

$$
\|(\mathbf{G}(u+v)-\mathbf{G}(u)) w\|_{\mathcal{X}} \leq \epsilon\|w\|_{\mathcal{X}} \leq \epsilon\|w\|_{\mathcal{W}} .
$$

Hence, if $\|v\|_{\mathcal{W}}<\delta$ then the operator norm of $\mathbf{G}(u+v)-\mathbf{G}(u)$ is less than $\epsilon$. This completes the proof.

We will later make use of some further properties of $\mathbf{F}$, as proved in the following lemma.

Lemma 2.4. Assuming that hypotheses (H-F1)-(H-F3) hold, the map $\mathbf{F}$ is bounded on the bounded subsets of $\mathcal{W}$ and is also weakly sequentially continuous.

Proof. Once again,

$$
\mathbf{F}(u)(t, x)=u(t, x) \int_{0}^{1} D_{z} F(t, x, s u(t, x)) \mathrm{d} s .
$$

Let $\mathcal{H}$ be a bounded subset of $\mathcal{W}$. Since $\mathcal{H}$ is bounded in the space $L^{\infty}((0, \infty) \times \Omega)$, there is $M>0$ such that $|u(t, x)|<M$ for each $u \in \mathcal{H}$ and $(t, x) \in[0, \infty) \times$ $\Omega$. By using Hypothesis (H-F2) with $J=(-M, M)$, we have $N>0$ such that $\left|D_{z} F(t, x, s u(t, x))\right|<N$ for all $(t, x) \in[0, \infty) \times \Omega$ and all $u \in \mathcal{H}$. We take $p^{t h}$ powers in (2.10) to find that $\|\mathbf{F}(u)(t, x)\|_{\mathcal{X}}<N\|u\|_{\mathcal{X}} \leq N\|u\|_{\mathcal{W}}$ for all $u \in \mathcal{H}$. This proves the first claim.

Now let $\left\{u_{n}\right\}$ be a sequence in $\mathcal{W}$ that converges weakly to some limit $u \in \mathcal{W}$. Since pointwise evaluations are continuous in $\mathcal{W}$, the convergence is also pointwise in $[0, \infty) \times \Omega$. In fact, the convergence is also uniform on the compact subsets of $[0, \infty) \times \Omega$. This is easily verified using the uniform equicontinuity of bounded subsets of $\mathcal{W}$. It now follows from Hypothesis $(\mathrm{H}-\mathrm{F} 2)$ that the sequence $\left\{\mathbf{F}\left(u_{n}\right)\right\}$ converges to $\mathbf{F}(u)$ uniformly on compact subsets of $[0, \infty) \times \Omega$. This sequence is also bounded in $\mathcal{X}=L^{p}((0, \infty) \times \Omega)$, by the first half of this lemma. It is readily verified that this results in the desired weak convergence of $\left\{\mathbf{F}\left(u_{n}\right)\right\}$ to $\mathbf{F}(u)$ in $L^{p}((0, \infty) \times \Omega)$. 
REMARK 2.5. Everything done to this point has an immediate and obvious extension to the whole line setting. That is, one can replace $[0, \infty)$ by $\mathbb{R}$ throughout this section. We will need to make use of this fact later. We will use the subscript $\mathbb{R}$ when discussing the resulting function spaces. For example, $\mathcal{X}_{\mathbb{R}}:=L^{p}\left(\mathbb{R}, L^{p}(\Omega)\right)$.

\section{The Fredholm property}

The goal of this short section is to prove that the Fredholm property and index of the $C^{1}$ operator $\Phi$ depend only on the Fredholm property of the linear operator $D \Phi(0)$. This is accomplished by proving that $D \Phi(u)-D \Phi(0)$ is compact for all $u \in \mathcal{W}$. We begin with a lemma that will be used here, and then again during the proof of Theorem 4.9 .

Lemma 3.1. Suppose that $g \in L^{\infty}((0, \infty) \times \Omega)$ is such that

$$
\lim _{T \rightarrow \infty}\|g\|_{L^{\infty}((T, \infty) \times \Omega)}=0 .
$$

Let $\left\{u_{n}\right\}$ be a bounded sequence in $W^{1, p}\left((0, \infty), L^{p}(\Omega)\right)$, and define $v_{n}(t, x):=$ $g(t, x) u_{n}(t, x)$. Then the sequence $\left\{v_{n}\right\}$ has a subsequence that converges in $\mathcal{X}=$ $L^{p}\left((0, \infty), L^{p}(\Omega)\right)$.

Proof. For each $N \in \mathbb{N}$, the space $W^{1, p}\left((0, N), L^{p}(\Omega)\right)$ is compactly contained in $L^{p}\left((0, N), L^{p}(\Omega)\right)$ (Simon [35, Theorem 1].) By a diagonal argument, there exists a subsequence $\left\{u_{n_{k}}\right\}$ of $\left\{u_{n}\right\}$ such that for all $N \in \mathbb{N}$, the sequence $\left\{\left.u_{n_{k}}\right|_{(0, N)}\right\}$ is Cauchy in $L^{p}\left((0, N), L^{p}(\Omega)\right)$.

To see that $\left\{v_{n_{k}}\right\}$ is the desired subsequence that is Cauchy (and hence convergent) in $\mathcal{X}$, let $\epsilon>0$. By (3.1) and the boundedness of $\left\{u_{n}\right\}$ in $\mathcal{X}$, there is $N \in \mathbb{N}$ such that for all $m, n \in \mathbb{N}$,

$$
\left\|v_{n}-v_{m}\right\|_{L^{p}\left((N, \infty), L^{p}(\Omega)\right)}<\epsilon / 2 .
$$

For sufficiently large $k$ and $j$ in $\mathbb{N}$,

$$
\left\|v_{n_{j}}-v_{n_{k}}\right\|_{L^{p}\left((0, N), L^{p}(\Omega)\right)}<\epsilon / 2
$$

as well. This completes the proof.

Let $E_{0}: \mathcal{W} \rightarrow \mathcal{W}(0)$ denote the evaluation map $u \mapsto u(0)$.

THEOREM 3.2. Continue to assume that $A$ and $F$ satisfy the hypotheses $(\mathrm{H})$. If $\left(\frac{\mathrm{d}}{\mathrm{d} t}-\mathbf{A}, E_{0}\right)$ is Fredholm of index zero from $\mathcal{W}$ into $\mathcal{Y}$, then the operator $\Phi$ is Fredholm of index zero from $\mathcal{W}$ into $\mathcal{Y}$.

Proof. Notice that because of equation (2.6) and hypothesis (H-F3), the derivative of $\Phi$ at $u=0$ is given by $D \Phi(0)=\left(\frac{\mathrm{d}}{\mathrm{d} t}-\mathbf{A}, E_{0}\right)$. In particular, $D \Phi(0)$ is Fredholm of index zero by assumption, and it now suffices to show that $D \Phi(u)-D \Phi(0)$ is compact for each $u \in \mathcal{W}$.

Fix $u \in \mathcal{W}$, and put $K:=D \Phi(u)-D \Phi(0)$. Let $\left\{v_{n}\right\}$ be a bounded sequence in $\mathcal{W}$. We show that $\left\{K v_{n}\right\}$ has a Cauchy subsequence in $\mathcal{X}$. First, note that $\left(K v_{n}\right)(t, x)=D_{z} F(t, x, u(t, x)) v_{n}(t, x)$. The norm $\|u(t, \cdot)\|_{X}$ tends to zero as $t \rightarrow \infty$, since $u \in W^{1, p}((0, \infty), X)$. According to Lemma 2.2, this implies that the maximum norm $\|u(t, \cdot)\|_{L^{\infty}(\Omega)}$ also tends to zero as $t \rightarrow \infty$. With hypotheses (H-F2) and (H-F3), this implies that $\left\|D_{z} F(t, \cdot, u(t, \cdot))\right\|_{L^{\infty}(\Omega)}$ tends to zero as $t \rightarrow \infty$. An application of Lemma 3.1 with $g(t, x)=D_{z} F(t, x, u(t, x))$ completes the proof. 
Remark 3.3. To ensure that $\left(\frac{\mathrm{d}}{\mathrm{d} t}-\mathbf{A}, E_{0}\right)$ is Fredholm of index zero from $\mathcal{W}$ onto $\mathcal{Y}$, it is enough to assume that $\frac{\mathrm{d}}{\mathrm{d} t}-\mathbf{A}$ is an isomorphism of $\mathcal{W}_{0}$ onto $\mathcal{X}$, where $\mathcal{W}_{0}:=\{u \in \mathcal{W} \mid u(0)=0\}$. Indeed, this is true if and only if $\left(\frac{\mathrm{d}}{\mathrm{d} t}-\mathbf{A}, E_{0}\right)$ is an isomorphism of $\mathcal{W}$ onto $\mathcal{Y}$, since ker $E_{0}=\mathcal{W}_{0} \cong \mathcal{W} / \mathcal{W}(0)$ and rge $E_{0}=\mathcal{W}(0)$. Of course, it is also enough to assume directly that $\left(\frac{\mathrm{d}}{\mathrm{d} t}-\mathbf{A}, E_{0}\right)$ is an isomorphism of $\mathcal{W}$ onto $\mathcal{Y}$.

\section{Properness on closed bounded subsets}

In this section, we establish a necessary and sufficient condition for $\Phi$ to be proper on the closed, bounded subsets of $\mathcal{W}$. The essential idea is the following. We are to show that $\left\{u_{n}\right\}$ has a convergent subsequence if $\left\{u_{n}\right\}$ is a bounded sequence in $\mathcal{W}$ such that $\Phi\left(u_{n}\right)$ converges. To find a convergent subsequence of $\left\{u_{n}\right\}$, we use a result from Rabier $[\mathbf{2 9}]$ that characterizes the relatively compact subsets of $C_{\{0\}}(\mathbb{R}, X)$, which is the Banach space of all continuous functions $u$ from $\mathbb{R}$ into $X$ such that $\lim _{|t| \rightarrow \infty}\|u(t)\|_{X}=0$. To do so, one must check the "equi-decay" of the sequence $\left\{u_{n}(t)\right\}$ in $X$ as $n \rightarrow \infty$.

This "equi-decay" is characterized in [29] by a condition involving sequences of the form $\left\{u_{n}\left(\cdot+\xi_{n}\right)\right\}$, where $\xi_{n} \rightarrow \infty$. It is this temporal translation to infinity that brings one to the condition that no equation of the form $u_{t}-\mathbf{B} u+\mathbf{G}(u)=0$ have a nontrivial solution $u \in \mathcal{W}_{\mathbb{R}}$, where $B$ and $G$ are any uniform-on-compacta limits of temporal translations of $A$ and $F$. It is an artifact of the translation to infinity that this condition involves problems on the whole line, even though the original problem is posed on the half line.

REMARK 4.1. In this section we will follow the following convention. Any vector valued function (in particular, any real valued function) that depends on a variable $t \geq 0$ is extended to negative values of $t$ whenever convenient, by using the even extension. Of course, this convention is only used where needed and never for a function whose domain already allows negative values of $t$.

For example, let $u \in \mathcal{W}$ and $\xi_{n} \rightarrow \infty$ in $\mathbb{R}$. Then the sequence $\left\{u\left(t+\xi_{n}, x\right)\right\}$ is well defined for all $t \in \mathbb{R}$ under this convention, and this sequence can hence have a well-defined pointwise or uniform-on-compacta limit on all of $\mathbb{R}$. Notice that this kind of sequence is locally eventually independent of the choice of extension (since $t+\xi_{n}$ is eventually positive), and so any limit function is independent of the choice of extension. This is characteristic of our later use of this "even extension convention", which will occur with minimal further comment. The purpose of this perhaps distracting convention is to avoid the alternate distraction of a prominently displayed extension operator.

4.1. Topological preliminaries. If $I$ is an unbounded interval, we follow [29] in using $C_{\{0\}}(I, X)$ to denote the Banach space of all continuous functions $u$ from $I$ into $X$ such that $\lim _{|t| \rightarrow \infty}\|u(t)\|_{X}=0$. Also, $C_{b}(I, X)$ denotes the larger Banach space of all bounded continuous functions from $I$ into $X$. Both spaces are normed by using $\|u\|=\sup _{t \in I}\|u(t)\|_{X}$. Also, if $\mathcal{H}$ is a subset of $C_{b}(I, X)$, then $\mathcal{H}(I)$ means the subset $\{u(t) \mid u \in \mathcal{H}, t \in I\}$ of $X$.

Lemma 4.2 (Rabier [29, Theorem 2]). A subset $\mathcal{H}$ of $C_{\{0\}}\left(\mathbb{R}, L^{p}(\Omega)\right)$ is relatively compact if and only if the following three conditions hold:

(i) The set $\mathcal{H}(\mathbb{R})$ is relatively compact in $L^{p}(\Omega)$. 
(ii) The set $\mathcal{H}$ is uniformly equicontinuous.

(iii) For all sequences $\left\{u_{n}\right\} \subset \mathcal{H}$ and $\left\{\xi_{n}\right\} \subset \mathbb{R}$, if $\left|\xi_{n}\right| \rightarrow \infty$ and there is some $\tilde{u} \in C_{b}\left(\mathbb{R}, L^{p}(\Omega)\right)$ such that

$$
\lim _{n \rightarrow \infty}\left\|u_{n}\left(t+\xi_{n}\right)-\tilde{u}(t)\right\|_{L^{p}(\Omega)}=0 \quad \forall t \in \mathbb{R},
$$

then $\tilde{u}=0$.

COROllary 4.3. A subset $\mathcal{H}$ of $C_{\{0\}}\left([0, \infty), L^{p}(\Omega)\right)$ is relatively compact if and only if the following three conditions hold:

(i) The set $\mathcal{H}([0, \infty))$ is relatively compact in $L^{p}(\Omega)$.

(ii) The set $\mathcal{H}$ is uniformly equicontinuous.

(iii) For all sequences $\left\{u_{n}\right\} \subset \mathcal{H}$ and $\left\{\xi_{n}\right\} \subset \mathbb{R}$, if $\xi_{n} \rightarrow \infty$ and there is some $\tilde{u} \in C_{b}\left(\mathbb{R}, L^{p}(\Omega)\right)$ such that ${ }^{2}$

$$
\lim _{n \rightarrow \infty}\left\|u_{n}\left(t+\xi_{n}\right)-\tilde{u}(t)\right\|_{L^{p}(\Omega)}=0 \quad \forall t \in \mathbb{R},
$$

then $\tilde{u}=0$.

Proof. Use the even extension of the elements of $C_{\{0\}}\left([0, \infty), L^{p}(\Omega)\right)$ to $\mathbb{R}$. Now apply Lemma 4.2.

REMARK 4.4. When using Lemma 4.2 or Corollary 4.3 , one often finds that the set $\mathcal{H}$ consists of the terms of a sequence $\left\{v_{n}\right\}$. If so, one may assume $\left\{u_{n}\right\}$ is a subsequence of $\left\{v_{n}\right\}$ when checking condition (iii).

Recall that the compact-open topology on a space of continuous functions on a metric space $M$ is characterized by uniform convergence on each compact subset of $M$. Accordingly, if $\left\{T_{n}\right\}$ is a sequence of continuous functions from $M$ into a metric space $N$, we will write $T=$ co- $\lim _{n \rightarrow \infty} T_{n}$ if the sequence $\left\{T_{n}\right\}$ converges uniformly to $T$ on each compact subset of $M$. In each use of this notation, $M$ and $N$ will be clear from context.

Lemma 4.5. Suppose that F satisfies Hypotheses $(\mathrm{H}-\mathrm{F} 1)-(\mathrm{H}-\mathrm{F} 3)$. Let $\left\{\xi_{n}\right\} \subset \mathbb{R}$ be a sequence such that $\xi_{n} \rightarrow \infty$ and put $G_{n}(t, x, z):=F\left(t+\xi_{n}, x, z\right)$. Then there exist a subsequence $\left\{G_{n_{k}}\right\}$ and a function $G: \mathbb{R} \times \Omega \times \mathbb{R} \rightarrow \mathbb{R}$ such that

(1) $G=\operatorname{co}^{-\lim _{k \rightarrow \infty}} G_{n_{k}}$,

(2) G satisfies Hypotheses $(\mathrm{H}-\mathrm{F} 1)-(\mathrm{H}-\mathrm{F} 3)$, but with $[0, \infty)$ replaced by $\mathbb{R}$ (and $F$ replaced by $G)$, and

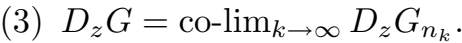

Proof. The classical Ascoli-Arzela Theorem applies for each compact subset $K$ of $\mathbb{R} \times \Omega \times \mathbb{R}$. (The boundedness of $\left\{G_{n}(\alpha) \mid n \in \mathbb{N}, \alpha \in K\right\}$ and and the equicontinuity of $\left\{\left.G_{n}\right|_{K}\right\}$ follow from hypothesis (H-F2).) Apply the AscoliArzela theorem recursively for an increasing sequence of compact sets that exhausts $\mathbb{R} \times \Omega \times \mathbb{R}$. This diagonal argument yields a function $G$ and a subsequence $\left\{G_{n_{k}}\right\}$ such that $G=\operatorname{co-} \lim _{k \rightarrow \infty} G_{n_{k}}$. Repeat this process with $\left\{D_{z} G_{n_{k}}\right\}$ to obtain a further subsequence (still denoted $\left\{G_{n_{k}}\right\}$ ) and a function $H$ such that $H=$ co- $\lim _{k \rightarrow \infty} D_{z} G_{n_{k}}$. As usual, since the convergence is uniform on compact sets, $D_{z} G$ exists and $D_{z} G=H$. Thus, $G$ satisfies Hypothesis (H-F1). It is easy to verify that Hypotheses (H-F2) and (H-F3) are inherited by $G$ from $F$.

\footnotetext{
${ }^{2}$ See Remark 4.1.
} 
Definition 4.6. Given a function $F$ that satisfies the hypotheses of Lemma 4.5, we define

$$
\omega(F)=\left\{G \mid G=\operatorname{co-} \lim G_{n} \text { for some sequence } \xi_{n} \rightarrow \infty\right\} .
$$

Here, $G_{n}(t, x, z):=G\left(t+\xi_{n}, x, z\right)$, just as in Lemma 4.5. It is a corollary of Lemma 4.5 that $\omega(F)$ is nonempty.

LEMMA 4.7. Let $\left\{\xi_{n}\right\} \subset \mathbb{R}$ be a sequence such that $\xi_{n} \rightarrow \infty$ and put $B_{n}(t):=$ $A\left(t+\xi_{n}\right)$. There exist a subsequence $\left\{B_{n_{k}}\right\}$ and a bounded, uniformly continuous function $B: \mathbb{R} \rightarrow \mathcal{L}(W, X)$ such that

$$
B=\underset{k \rightarrow \infty}{\operatorname{co-} \lim } B_{n_{k}} .
$$

Proof. The proof is similar to that of Lemma 4.5, and is omitted.

We define $\omega(A)$ in the same way as $\omega(F)$ :

Definition 4.8. Given a function $A$ that satisfies the hypotheses of Lemma 4.7, we define

$$
\omega(A)=\left\{B \mid B=\text { co-lim } B_{n} \text { for some sequence } \xi_{n} \rightarrow \infty\right\} .
$$

Notice that according to Lemma $4.7, \omega(A)$ is nonempty.

\subsection{A condition for properness.}

THEOREM 4.9. Assume that $A$ and $F$ continue to satisfy the hypotheses $(\mathrm{H})$, and also assume that $D \Phi(0)$ is semi-Fredholm ${ }^{3}$ from $\mathcal{W}$ into $\mathcal{Y}$. In addition, suppose that for every $B \in \omega(A)$ and $G \in \omega(F)$, the equation $\frac{\mathrm{d} u}{\mathrm{~d} t}-\mathbf{B} u+\mathbf{G}(u)=0$ has no nontrivial solution $u \in \mathcal{W}_{\mathbb{R}}$. Then $\Phi$ is proper on the closed bounded subsets of $\mathcal{W}$.

Proof. We are to show that $\left\{u_{n}\right\}$ has a subsequence convergent in $\mathcal{W}$ whenever $\left\{u_{n}\right\}$ is a bounded sequence in $\mathcal{W}$ such that $\left\{\Phi\left(u_{n}\right)\right\}$ is convergent in $\mathcal{Y}$. Let $\left\{u_{n}\right\}$ be so, and take $\left(f_{n}, g_{n}\right):=\Phi\left(u_{n}\right)$ and $(f, g):=\lim _{n \rightarrow \infty}\left(f_{n}, g_{n}\right)$. In particular, $\lim _{n \rightarrow \infty}\left(\frac{\mathrm{d} u_{n}}{\mathrm{~d} t}-\mathbf{A} u_{n}+\mathbf{F}\left(u_{n}\right)\right)=f$ in $\mathcal{X}$.

We begin by using Corollary 4.3 with $\mathcal{H}:=\left\{u_{n} \mid n \in \mathbb{N}\right\}$ to show that a subsequence of $\left\{u_{n}\right\}$ converges to a limit $u$ in $C_{\{0\}}\left([0, \infty), L^{p}(\Omega)\right)$. First, suppose for contradiction that $\mathcal{H}([0, \infty))$ is not relatively compact in $L^{p}(\Omega)$. Then there are a sequence $\left\{\xi_{k}\right\}$ in $[0, \infty)$ and a subsequence $\left\{u_{n_{k}}\right\}$ such that $\left\{u_{n_{k}}\left(\xi_{k}\right)\right\}$ has no subsequence that converges in $L^{p}(\Omega)$. Take $v_{k}:=u_{n_{k}}\left(\cdot+\xi_{k}\right)$, which is a bounded sequence in the space $W^{1, p}\left((0,1), L^{p}(\Omega)\right)$. Then $\left\{v_{k}(0)\right\}$ has no convergent subsequence in $L^{p}(\Omega)$. This contradicts the compact inclusion (see Simon $[\mathbf{3 5}]$ ) of the space $W^{1, p}\left((0,1), L^{p}(\Omega)\right)$ in $C\left([0,1], L^{p}(\Omega)\right)$.

Second, the uniform equicontinuity of $\mathcal{H}$ is a consequence of the boundedness of $\left\{u_{n}\right\}$ in the Sobolev space $W^{1, p}((0, \infty), X)$, using the usual Hölder embedding (recall $p>d+1>1$ ).

To verify the third condition in Corollary 4.3, suppose that a sequence $\left\{\xi_{k}\right\}$ of real numbers and a subsequence $\left\{u_{n_{k}}\right\}$ of $\left\{u_{n}\right\}$ are such that $\xi_{k} \rightarrow \infty$ and for some $\tilde{u} \in C_{b}\left(\mathbb{R}, L^{p}(\Omega)\right)$, the sequence $\left\{v_{k}\right\}$ is $L^{p}(\Omega)$-convergent to $\tilde{u}$ pointwise on $\mathbb{R}$, where $v_{k}(t):=u_{n_{k}}\left(t+\xi_{k}\right)$. Because $\left\{v_{k}\right\}$ is bounded in the reflexive Banach space $\mathcal{W}_{\mathbb{R}}$, we may assume with no loss of generality that $\left\{v_{k}\right\}$ has a $\mathcal{W}_{\mathbb{R}}$-weak limit

\footnotetext{
${ }^{3}$ This means that $\operatorname{dim} \operatorname{ker} D \Phi(0)<\infty$ and that rge $D \Phi(0)$ is closed in $\mathcal{Y}$. Of course, it is enough to suppose that $D \Phi(0)$ is an isomorphism of $\mathcal{W}$ onto $\mathcal{Y}$.
} 
$\hat{u}$. The continuity of each evaluation map $u \in \mathcal{W}_{\mathbb{R}} \mapsto u(t) \in X$ implies that $\tilde{u}=\hat{u}$. In particular, $\tilde{u} \in \mathcal{W}_{\mathbb{R}}$.

Let $G_{k}(t, x, z):=F\left(t+\xi_{k}, x, z\right)$ and $B_{k}(t):=A\left(t+\xi_{k}\right)$. By passing to subsequences (and relabeling) there are limit points $G:=\operatorname{co}^{-} \lim _{k \rightarrow \infty} G_{k}$ in $\omega(F)$ and $B:=\operatorname{co}^{-} \lim _{k \rightarrow \infty} B_{k}$ in $\omega(A)$; these are obtained by use of Lemmas 4.5 and 4.7. We will now examine the behavior of $\left\{f_{n_{k}}\left(\cdot+\xi_{k}\right)\right\}$ as $k \rightarrow \infty$. We have

$$
f_{n_{k}}\left(\cdot+\xi_{k}\right)=\frac{\mathrm{d} v_{k}}{\mathrm{~d} t}-\mathbf{B}_{\mathbf{k}} v_{k}+\mathbf{G}_{\mathbf{k}}\left(v_{k}\right)
$$

By the weak sequential continuity of bounded linear operators, the sequence $\left\{\frac{\mathrm{d} v_{k}}{\mathrm{~d} t}\right\}$ converges weakly to $\frac{\mathrm{d} \tilde{u}}{\mathrm{~d} t}$ in $\mathcal{X}_{\mathbb{R}}$. We will show that the sequence $\left\{\mathbf{B}_{\mathbf{k}} v_{k}\right\}$ converges weakly to $\mathbf{B} \tilde{u}$ in $\mathcal{X}_{\mathbb{R}}$. On one hand,

$$
\mathbf{B}\left(v_{k}-\tilde{u}\right) \stackrel{\mathrm{w}}{\longrightarrow} 0
$$

in $\mathcal{X}_{\mathbb{R}}$, again by weak sequential continuity. On the other hand, for each $t \in \mathbb{R}$

$$
\left\|\left(B_{k}(t)-B(t)\right) v_{k}(t)\right\|_{X} \leq\left\|B_{k}(t)-B(t)\right\|_{\mathcal{L}(W, X)}\left\|v_{k}(t)\right\|_{W} .
$$

Since the sequence $\left\{v_{k}\right\}$ is bounded in $L^{p}(\mathbb{R}, W)$, and $\left\{B_{k}\right\}$ converges to $B$ uniformly on compact intervals, it follows that $\left\{\left(\mathbf{B}_{\mathbf{k}}-\mathbf{B}\right) v_{k}\right\}$ converges in norm to zero in $L^{p}((-T, T) \times \Omega)$ for each $T>0$. Since $\left\{\left(\mathbf{B}_{\mathbf{k}}-\mathbf{B}\right) v_{k}\right\}$ is also bounded in $L^{p}(\mathbb{R} \times \Omega)$, one can readily verify that $\left(\mathbf{B}_{\mathbf{k}}-\mathbf{B}\right) v_{k} \stackrel{\mathrm{w}}{\rightarrow} 0$ in $\mathcal{X}_{\mathbb{R}}$. With (4.4), we conclude that

$$
\mathbf{B}_{\mathbf{k}} v_{k} \stackrel{\mathrm{w}}{\rightarrow} \mathbf{B} \tilde{u} \text { in } \mathcal{X}_{\mathbb{R}} .
$$

We next show that the sequence $\mathbf{G}_{\mathbf{k}}\left(v_{k}\right) \stackrel{\mathrm{w}}{\longrightarrow} \mathbf{G}(\tilde{u})$ weakly in $\mathcal{X}_{\mathbb{R}}$. Firstly,

$$
\mathbf{G}\left(v_{k}\right)-\mathbf{G}(\tilde{u}) \stackrel{\mathrm{w}}{\longrightarrow} 0 \quad \text { in } \mathcal{X}_{\mathbb{R}}
$$

since $\mathbf{G}$ is weakly sequentially continuous (see Lemma 2.4 and Remark 2.5). The sequence $\left\{v_{k}\right\}$ is bounded in $\mathcal{W}_{\mathbb{R}}$, and hence $\left\{v_{k}(t, x)\right\}$ is bounded uniformly on $\mathbb{R} \times \Omega$. Since $G=$ co-lim $_{k \rightarrow \infty} G_{k}$, it follows that $\mathbf{G}_{\mathbf{k}}\left(v_{k}\right)-\mathbf{G}\left(v_{k}\right) \rightarrow 0$ uniformly on compact subsets of $\mathbb{R} \times \Omega$. Moreover, the sequence $\left\{\mathbf{G}_{\mathbf{k}}\left(v_{k}\right)-\mathbf{G}\left(v_{k}\right)\right\}$ is also bounded in $L^{p}(\mathbb{R} \times \Omega)$. This follows from Lemma 2.4 and Remark 2.5, since $L^{p}$ norms are translation invariant. It is now straightforward to verify that $\mathbf{G}_{\mathbf{k}}\left(v_{k}\right)-$ $\mathbf{G}\left(v_{k}\right) \stackrel{\text { w }}{\rightarrow} 0$ in $\mathcal{X}_{\mathbb{R}}$. With (4.6), this implies that

$$
\mathbf{G}_{\mathbf{k}}\left(v_{k}\right)-\mathbf{G}(\tilde{u}) \stackrel{\mathrm{w}}{\longrightarrow} 0 \text { in } \mathcal{X}_{\mathbb{R}} .
$$

We have now treated all terms on the right side of (4.3), and we conclude from (4.5) and (4.7) that

$$
f_{n_{k}}\left(\cdot+\xi_{k}\right) \stackrel{\mathrm{w}}{\longrightarrow} \frac{\mathrm{d} \tilde{u}}{\mathrm{~d} t}-\mathbf{B} \tilde{u}+\mathbf{G}(\tilde{u}) \quad \text { in } \mathcal{X}_{\mathbb{R}} .
$$

On the other hand, it was assumed from the outset that $\left\{f_{n}\right\}$ is norm-convergent in $L^{p}(\mathbb{R} \times \Omega)$. This readily implies (since $\xi_{k} \rightarrow \infty$ ) that $\left\{f_{n_{k}}\left(\cdot+\xi_{k}\right)\right\}$ converges weakly to zero in $L^{p}(\mathbb{R} \times \Omega)$. With (4.8), we have proved that

$$
\frac{\mathrm{d} \tilde{u}}{\mathrm{~d} t}-\mathbf{B} \tilde{u}+\mathbf{G}(\tilde{u})=0 .
$$

By hypothesis, this implies that $\tilde{u}=0$. This completes the verification of the third condition in Corollary 4.3. Thus, the sequence $\left\{u_{n}\right\}$ possesses a subsequence $\left\{u_{n_{k}}\right\}$ that converges in $C_{\{0\}}\left([0, \infty), L^{p}(\Omega)\right)$ to a limit $u$. According to Lemma 2.2, it follows that $\left\{u_{n_{k}}(t, x)\right\}$ also converges to $u$ uniformly on $[0, \infty) \times \Omega$. By the 
reflexivity of $\mathcal{W}$, we may pass to a further subsequence (still denoted $\left\{u_{n_{k}}\right\}$ ) to obtain a weak limit $\hat{u} \in \mathcal{W}$ for $\left\{u_{n_{k}}\right\}$. Since pointwise evaluations are continuous in $\mathcal{W}$, this implies that $u=\hat{u} \in \mathcal{W}$. By the mean value theorem,

$$
\begin{aligned}
F\left(t, x, u_{n_{k}}(t, x)\right)-F(t, x, u(t, x)) & \\
= & D_{z} F\left(t, x, p_{n_{k}}(t, x)\right)\left(u_{n_{k}}(t, x)-u(t, x)\right) \\
= & \left(D_{z} F\left(t, x, p_{n_{k}}(t, x)\right)-D_{z} F(t, x, u(t, x))\right) . \\
& \quad \cdot\left(u_{n_{k}}(t, x)-u(t, x)\right) \\
& \quad+D_{z} F(t, x, u(t, x))\left(u_{n_{k}}(t, x)-u(t, x)\right)
\end{aligned}
$$

where $p_{n_{k}}$ lies between $u_{n_{k}}$ and $u$ pointwise. The uniform convergence of $\left\{u_{n_{k}}\right\}$ to $u$, along with hypotheses (H-F2) and (H-F3) imply that

$$
D_{z} F\left(t, x, p_{n_{k}}(t, x)\right) \rightarrow D_{z} F(t, x, u(t, x))
$$

uniformly on $[0, \infty) \times \Omega$. This causes the first term on the far right side of $(4.9)$ to tend to zero in $L^{p}(\mathbb{R} \times \Omega)$ as $k \rightarrow \infty$. By Lemma 3.1 with $g(t, x)=D_{z} F(t, x, u(t, x))$ there is a further subsequence (again denoted $\left\{u_{n_{k}}\right\}$ ) such that the second term on the far right side of (4.9) converges to zero in $L^{p}(\mathbb{R} \times \Omega)$. Altogether, we conclude that $\left\{\mathbf{F}\left(u_{n_{k}}\right)\right\}$ converges to $\mathbf{F}(u)$ in $L^{p}(\mathbb{R} \times \Omega)$.

As a result, $\lim _{n \rightarrow \infty}\left(\frac{\mathrm{d} u_{n}}{\mathrm{~d} t}-\mathbf{A} u_{n}\right)=-\mathbf{F}(u)+f$. This implies that $\left\{D \Phi(0) u_{n}\right\}$ converges to $(-\mathbf{F}(u)+f, g)$. According to Yood's criterion (see Deimling [6, Proposition 9.3]), the semi-Fredholm operator $D \Phi(0)$ is proper on the closed, bounded subsets of $\mathcal{W}$. This implies the existence of the desired $\mathcal{W}$-convergent subsequence of $\left\{u_{n}\right\}$.

REMARK 4.10. We note the similarity here in both the method and the results with those of Rabier and Stuart in $[\mathbf{3 1}]$ and [30]. Both of those papers deal with ordinary differential equations on the half line. The former focuses on autonomous problems, and the latter treats problems that have a single "limit" as $t \rightarrow \infty$ (that is, $\omega(A)=\left\{A_{0}\right\}$ and $\left.\omega(F)=\left\{F_{0}\right\}\right)$. The author first treated this more general case in his $\mathrm{PhD}$ thesis [22], for both ODEs and PDEs.

REMARK 4.11. Although we will not use this fact, the converse of Theorem 4.9 is true. That is, if one assumes instead that there are $B \in \omega(A)$ and $G \in \omega(F)$ such that $\frac{\mathrm{d} u}{\mathrm{~d} t}-\mathbf{B} u+\mathbf{G}=0$ for some nonzero $u \in \mathcal{W}_{\mathbb{R}}$, one can show that $\left\{\Phi\left(u\left(\cdot-\xi_{n}\right)\right)\right\}$ converges to zero in $\mathcal{Y}$ and also that $\left\{u\left(\cdot-\xi_{n}\right)\right\}$ has no $\mathcal{W}$-convergent subsequence.

\section{Existence of solutions by degree theory}

Definition 5.1. Let $f \in X$ and $g \in \mathcal{W}(0)$ be given. We say that " $f$ and $g$ satisfy the a priori bound condition (with respect to $A$ and $F$ )" when there is some $R>0$ such that the following condition holds. For some $C^{1}$ path $(q, r)=$ $\left(q_{s}, r_{s}\right):[0,1] \rightarrow X \times \mathcal{W}(0)$ with $\left(q_{0}, r_{0}\right)=(0,0)$ and $\left(q_{1}, r_{1}\right)=(f, g)$, all solutions $u \in \mathcal{W}$ to systems

$$
\begin{aligned}
u_{t}-A(t) u+F(t, x, u) & =q_{s}(t, x) ; \\
u(0, x) & =r_{s}(x)
\end{aligned}
$$

with $0 \leq s \leq 1$ satisfy $\|u\|_{\mathcal{W}} \leq R$. (That is, the preimage under $\Phi$ of some smooth path connecting the origin to $(f, g)$ is bounded in $\mathcal{W}$.) 
Remark 5.2. In Definition 5.1 it is certainly enough to use the linear path $\left(q_{s}, r_{s}\right)=(s f, s g)$. Indeed, it is enough to find a bound $R$ that depends only on some norm of $\left(q_{s}, r_{s}\right)$, for then any norm decreasing path will work. This is the approach that we take in Section 6.

We are now ready to prove our main result.

THEOREM 5.3. Assume that $A$ and $F$ continue to satisfy the hypotheses $(\mathrm{H})$, and also that:

(1) For all $h \in \mathcal{X}=L^{p}((0, \infty) \times \Omega)$, there is exactly one $u \in \mathcal{W}$ such that $u(0, \cdot)=0$ in $\Omega$ and $u_{t}-A(t) u=h(t, x)$.

(2) There is no nontrivial solution $u \in \mathcal{W}$ to the nonlinear homogeneous initial value problem

$$
\begin{aligned}
u_{t}-A(t) u+F(t, x, u) & =0, \\
u(0, x) & =0 .
\end{aligned}
$$

(3) For all $B \in \omega(A)$ and all $G \in \omega(F)$, there is no nontrivial solution $u \in \mathcal{W}_{\mathbb{R}}$ to

$$
u_{t}-B(t) u+G(t, x, u)=0 .
$$

(4) The functions $f \in \mathcal{X}$ and $g \in \mathcal{W}(0)$ satisfy the a priori bound condition. Then there is a solution $u \in \mathcal{W}$ to the initial value problem

$$
\begin{aligned}
u_{t}-A(t) u+F(t, x, u) & =f(t, x), \\
u(0, x) & =g(x) .
\end{aligned}
$$

Proof. Let $R>0$ be the a priori bound that is assumed to exist for solutions along a $C^{1}$ path $\left(q_{s}, r_{s}\right), 0 \leq s \leq 1$ joining $(0,0)$ to $(f, g)$. Let $B$ be the open ball of radius $R+1$ centered at the origin of $\mathcal{W}$. According to Lemma 2.3 and the hypotheses $(\mathrm{H})$, the operator $\Phi$ is in $C^{1}(\mathcal{W}, \mathcal{Y})$. Assumption (1), along with Theorem 3.2 and Remark 3.3, imply that $\Phi$ is a Fredholm map of index zero. This, along with assumption (3) and Theorem 4.9 imply that $\left.\Phi\right|_{\bar{B}}$ is proper. All of this ensures that $\Phi$ is $B$-admissible, in the sense of Definition 4.1 of [24].

The a priori bound condition ensures that $\left(q_{s}, r_{s}\right) \in \mathcal{Y} \backslash \Phi(\partial B)$ for all $0 \leq s \leq 1$. As introduced following Corollary 5.5 of [24], the absolute degree $|d|\left(\Phi, B,\left(q_{s}, r_{s}\right)\right)$ is hence a well defined nonnegative integer for all $0 \leq s \leq 1$. Introduce the homotopy $h:[0,1] \times \mathcal{W} \rightarrow \mathcal{Y}$ by

$$
h(s, u):=\Phi(u)-\left(q_{s}, r_{s}\right) .
$$

We must verify that $h$ is a $B$-admissible homotopy, in the sense of Definition 4.2 of [24]. It is clear that $h$ is $C^{1}$. To see that $h$ is Fredholm of index one, note that $D h(s, u)$ is a rank one perturbation of the linear map $L:=(0, D \Phi(u)): \mathbb{R} \times \mathcal{W} \rightarrow \mathcal{Y}$. The map $L$ has the same range and target space as $D \Phi(u)$, but $\operatorname{ker} L=\mathbb{R} \times$ ker $D \Phi(u)$. Thus, $L$ is Fredholm of index one because $D \Phi(u)$ is Fredholm of index zero. The compact perturbation $D h(s, u)$ of $L$ therefore enjoys the same Fredholm property and index.

The last requirement for $h$ to be a $B$-admissible homotopy is that $h_{[0,1] \times \bar{B}}$ should be proper. This readily follows from the properness of $\left.\Phi\right|_{\bar{B}}$, taking into account the compactness of $[0,1]$ and the continuity of the path $(q, r)$. It now 
follows from Theorem 5.1 of $[\mathbf{2 4}]$ that $|d|(h(s, \cdot), B,(0,0))$ is independent of $0 \leq$ $s \leq 1$.

Now, assumption (2) means that $\Phi^{-1}((0,0))=\{0\}$, and thus assumption (1) suffices to ensure that $(0,0)$ is a regular point of $\left.\Phi\right|_{B}$. Thus, according to the definition of the degree at regular values of $\Phi,|d|(\Phi, B,(0,0))=1$. Therefore, $|d|(\Phi-(f, g), B,(0,0))=1 \neq 0$ as well, since $h(0, \cdot)=\Phi$ and $h(1, \cdot)=\Phi-(f, g)$. It follows from the normalization property of the degree that $\Phi(u)-(f, g)=0$ for some $u \in \mathcal{W}$. This completes the proof.

REMARK 5.4. In general, some smoothness of $\partial \Omega$ is required to satisfy hypothesis (1) of Theorem 5.3. For example, $\partial \Omega$ should be $C^{2}$, or at least $C^{1,1}$. Otherwise the solution $u$ to $u_{t}-A(t) u=f$ is not necessarily in $W^{1,2}$, even if $A(t)=\Delta$. See $[\mathbf{1 7}$, Section 7.5$]$ for details.

REMARK 5.5. We note that the hypothesis (3) of Theorem 5.3 does not rule out nontrivial steady state or periodic solutions to the hull equations. Only solutions $u \in \mathcal{W}_{\mathbb{R}} \backslash\{0\}$ are prohibited.

REMARK 5.6. By Borsuk's theorem, one may replace hypotheses (1) and (2) of Theorem 5.3 with the assumptions that $D \Phi(0)$ is Fredholm of index zero from $\mathcal{W}$ into $\mathcal{Y}$ and that $F$ is odd in the third argument.

\section{Application to semilinear parabolic problems}

We will apply Theorem 5.3 to a more particular problem. Let $(A(t))_{t \geq 0}$ be a family of uniformly elliptic operators in divergence form:

$$
-A(t) u=-\frac{\partial}{\partial x_{j}}\left(a^{i j} \frac{\partial u}{\partial x_{i}}\right)+b^{i} \frac{\partial u}{\partial x_{i}}+c u,
$$

where we are following the Einstein summation convention; any term with a repeated index is summed over that index from 1 to $d$. Let $\tilde{b}^{i}:=b^{i}-\frac{\partial}{\partial x_{j}} a^{i j}$ denote the first order coefficients for the non-divergence form of $A$. The functions $a^{i j}$, $\tilde{b}^{i}$, and $c$ are all assumed to be bounded and uniformly continuous functions from $[0, \infty) \times \Omega$ into $\mathbb{R}$, in accordance with $(\mathrm{H}-\mathrm{A} 1) .{ }^{4}$ Of course, that $A$ is uniformly elliptic means that the matrix $\left(a^{i j}\right)$ is symmetric and that for some $\theta>0$,

$$
a^{i j}(t, x) \xi_{j} \xi_{i} \geq \theta|\xi|^{2}, \quad \forall \xi \in \mathbb{R}^{d}, \forall(t, x) \in[0, \infty) \times \Omega .
$$

We now introduce three additional assumptions on $A$, which we first list and then briefly discuss. First,

$$
\text { the map } \frac{\mathrm{d}}{\mathrm{d} t}-\mathbf{A} \text { is an isomorphism of } \mathcal{W}_{0} \text { onto } \mathcal{X} \text {. }
$$

Second, there is a constant $C>0$ such that for all $t \geq 0$ and all $u \in W^{2, p}(\Omega) \cap$ $W_{0}^{1, p}(\Omega)$,

$$
-\int_{\Omega}(A(t) u) u|u|^{p-2} \mathrm{~d} x \geq C\|u\|_{L^{p}(\Omega)}^{p} .
$$

Third, for all $i, j=1, \ldots, d$

$$
c \geq 0 \text { and } a^{i j}, \tilde{b}^{i} \in C^{0,(d+1) / p}(\overline{(0 \infty) \times \Omega}) .
$$

${ }^{4}$ For most of the sequel we could do without this continuity in $x$. However, see condition (H-A4) below. 
REMARK 6.1. The assumption (H-A2) is simply that item (1) in Theorem 5.3 should hold. A list of explicit and general conditions under which (H-A2) holds is beyond the scope of this paper. For such a discussion, we refer the reader to $[\mathbf{2 8}$, Section 7] and the references therein, which we now use to exemplify some conditions under which (H-A2) holds. Assume there are functions $a_{0}^{i j}, b_{0}^{i}$, and $c_{0}$ of $x$ such that

$$
\lim _{t \rightarrow \infty} a^{i j}(t, x)=a_{0}^{i j}(x), \quad \lim _{t \rightarrow \infty} b^{i}(t, x)=b_{0}^{i}(x), \quad \text { and } \quad \lim _{t \rightarrow \infty} c(t, x)=c_{0}(x)
$$

uniformly in $x$. If $c(t, x)$ is uniformly sufficiently large, then (H-A2) holds under familiar regularity assumptions (with respect to $x$ ) about the coefficients of $A$ and $\partial \Omega$. The main idea in [28, Section 7$]$ is to check that a particular technical condition for $A(t)$ hold for each $t$. Therefore, it is also helpful to consult [27, Section 5], which discusses this condition in the autonomous setting. In particular, if $a^{i j}=\delta^{i j}$ and $b^{i}=0$ then (H-A2) holds if $-\tilde{c}$ is a real constant not in the spectrum of the Laplacian $\Delta$. To be more particular, (H-A2) holds in case $A(t) u=\Delta u$. To our knowledge, the results in Theorem 6.13 are new even in this case.

REMARK 6.2. The condition (H-A3) requires that the family $(A(t))_{t \geq 0}$ be strictly dissipative, uniformly with respect to $t \geq 0$. We use (H-A3) in the proof of Lemma 6.7 to obtain a priori bounds on the $L^{p}$ norms of solutions. We will verify as Lemma 6.5 that $A$ satisfies this assumption as long as the first order terms are not too large and the zeroth order term is uniformly large enough.

REMARK 6.3. The hypothesis (H-A4) introduces a Hölder continuity condition to the first and second order coefficients in (the non-divergence form of) $A$. This is used to facilitate a convolution method to obtain a priori bounds on the $L^{\infty}$ norms of solutions in the proof of Lemma 6.10. This is the first and only place in this paper where Hölder continuity is assumed. Condition (H-A4) allows for any given order of Hölder continuity, as long as one is willing to increase the value of $p$ accordingly. Notice also that it suffices to have a Hölder condition of exponent $\lambda>d /(p+1)($ see $[\mathbf{1}$, Theorem 1.34]) .

The assumption that $c \geq 0$ is to ensure that $-\mathbf{A} u$ is nonnegative at a local maximum of $u$, for smooth $u$. This is used in the proof of Lemma 6.10, and so the assumption is included in (H-A4).

For the nonlinearity, we take $F$ to be any function that satisfies the hypotheses (H-F1)-(H-F3), along with the following additional conditions:

$$
\begin{gathered}
F(t, x, z) z \geq 0, \quad \forall(t, x, z) \in[0, \infty) \times \Omega \times \mathbb{R}, \text { and } \\
\lim _{|z| \rightarrow \infty}|F(t, x, z)|=\infty, \quad \text { uniformly in }(t, x) .
\end{gathered}
$$

The hypothesis (H-F4) is for dissipativity and helps to find bounds in $L^{p}$. Condition (H-F5) will help to find bounds in $L^{\infty}$, because (H-F5) reduces the problem of bounding $u$ to that of bounding $F(t, x, u)$. Assumption (H-F5) can be weakened, as will be explained in Remark 6.11.

Our largest obstacle in applying Theorem 5.3 will be to find a priori bounds on solutions (item (4) of Theorem 5.3). We first find a priori bounds in $L^{p}((0, \infty), \Omega)$ and then in $L^{\infty}((0, \infty), \Omega)$. It is then easy to use these bounds along with (H-A2) to find bounds in $\mathcal{W}$. Also, the bounds in $L^{p}$ allow us to verify item (3) of Theorem 5.3 (properness). 
To begin, we prove Lemma 6.5 concerning the practical verification of (H-A3). To do so, we use a variant of the Poincaré inequality, a proof of which the author was unable to find in the literature:

LEMma 6.4. There is a constant $C_{p, \Omega}$ such that

$$
\int_{\Omega}|u|^{p} \mathrm{~d} x \leq C_{p, \Omega} \int_{\Omega}|u|^{p-2}|\nabla u|^{2} \mathrm{~d} x
$$

whenever $u \in W_{0}^{1, p}(\Omega)$.

Proof. Recall that $p>d+1 \geq 2$. The proof is similar to that of the classical Poincaré inequality, and is only sketched. Without loss of generality, $\Omega$ is a rectangle $\Pi_{i=1}^{d}\left(a_{i}, b_{i}\right)$. First,

$$
|u(x)|^{p}=\int_{a_{1}}^{x_{1}} \frac{\partial}{\partial \xi}\left|u\left(\xi, x^{\prime}\right)\right|^{p} \mathrm{~d} \xi \leq p \int_{a_{1}}^{b_{1}}\left|u\left(\xi, x^{\prime}\right)\right|^{p-1}\left|\frac{\partial u}{\partial x_{1}}\left(\xi, x^{\prime}\right)\right| \mathrm{d} \xi .
$$

Now integrate both sides, first with respect to $x_{1}$, and then with respect to the remaining variables $x^{\prime}$. The result is

$$
\int_{\Omega}|u(x)|^{p} \mathrm{~d} x \leq p\left(b_{1}-a_{1}\right) \int_{\Omega}|u(x)|^{p-1}\left|\frac{\partial u}{\partial x_{1}}\right| \mathrm{d} x .
$$

With use of the Cauchy-Schwartz inequality, it follows that

$$
\int_{\Omega}|u(x)|^{p} \mathrm{~d} x \leq p\left(b_{1}-a_{1}\right)\left(\int_{\Omega}|u(x)|^{p} \mathrm{~d} x\right)^{1 / 2}\left(|u(x)|^{p-2}\left|\frac{\partial u}{\partial x_{1}}\right|^{2} \mathrm{~d} x\right)^{1 / 2} .
$$

The desired result follows upon squaring both sides.

LEMMA 6.5. Let

$$
c^{\mathrm{inf}}=\inf \{|c(t, x)| \mid(t, x) \in[0, \infty) \times \Omega\}
$$

and let $b^{\infty}=\max _{i=1}^{d}\left\|b^{i}\right\|_{\infty}$. Condition (H-A3) holds if

$$
(p-1) \theta-\epsilon b^{\infty} \geq 0
$$

and

$$
C_{p, \Omega}\left((p-1) \theta-\epsilon b^{\infty}\right)-\frac{b^{\infty}}{4 \epsilon}+c^{\mathrm{inf}}>0
$$

both hold for some $\epsilon>0$.

PROOF. Using integration by parts and the ellipticity of $A$,

$$
\begin{aligned}
-\int_{\Omega} \frac{\partial}{\partial x_{j}}\left(a^{i j} \frac{\partial u}{\partial x_{i}}\right) u|u|^{p-2} \mathrm{~d} x & =(p-1) \int_{\Omega} a^{i j} \frac{\partial u}{\partial x_{i}} \frac{\partial u}{\partial x_{j}}|u|^{p-2} \mathrm{~d} x \\
& \geq(p-1) \theta \int_{\Omega}|\nabla u|^{2}|u|^{p-2} \mathrm{~d} x .
\end{aligned}
$$

For the first order terms, we use Cauchy's inequality with epsilon:

$$
\int b^{i} \frac{\partial u}{\partial x_{i}} u|u|^{p-2} \mathrm{~d} x \geq-b^{\infty} \int_{\Omega} \epsilon|\nabla u|^{2}|u|^{p-2}+\frac{1}{4 \epsilon}|u|^{p} \mathrm{~d} x
$$


Since $\int c|u|^{p} \geq c^{\inf } \int|u|^{p}$, it follows that

$$
\begin{aligned}
-\int_{\Omega}(A(t) u) u u|u|^{p} \mathrm{~d} x \geq\left((p-1) \theta-\epsilon b^{\infty}\right) \int_{\Omega}|\nabla u|^{2}|u|^{p-2} \mathrm{~d} x & \\
& +\left(c^{\inf }-\frac{b^{\infty}}{4 \epsilon}\right) \int_{\Omega}|u|^{p} \mathrm{~d} x .
\end{aligned}
$$

According to (6.1), the inequality is preserved when we apply the $p$-Poincaré inequality to the first integral on the right side of (6.3). The result is

$$
-\int_{\Omega}(A(t) u) u u|u|^{p} \mathrm{~d} x \geq\left(C_{p, \Omega}\left((p-1) \theta-\epsilon b^{\infty}\right)-\frac{b^{\infty}}{4 \epsilon}+c^{\text {inf }}\right) \int_{\Omega}|u|^{p} \mathrm{~d} x .
$$

Because of (6.2), this verifies (H-A3).

COROLlary 6.6. Condition (H-A3) holds if $c^{\mathrm{inf}}$ is sufficiently large with respect to $\theta, C_{p, \Omega}$, and $b^{\infty}$. In particular, condition (H-A3) holds if $b_{i}=0$ for each $i=1, \ldots, d$, and $c(t, x) \geq 0$.

6.1. Bounds in $\mathbf{L}^{\mathbf{p}}$. We use an energy method to obtain a priori bounds for the $L^{p}$ norm of solutions.

Lemma 6.7. Let $A$ satisfy hypotheses (H-A1) and (H-A3), and let $F$ satisfy Hypotheses (H-F1)-(H-F4). Let $R>0$. There exists a constant $C_{p}=C_{p}(R)>0$ such that $\|u\|_{p} \leq C$ whenever $u \in \mathcal{W}$ with $\left\|u_{t}-\mathbf{A} u+\mathbf{F}(u)\right\|_{p} \leq R$ and $\|u(0)\|_{\mathcal{W}(0)} \leq R$.

Proof. Put $f:=u_{t}-\mathbf{A} u+\mathbf{F}(u)$ and $g:=u(0)$. For each $t \geq 0$, we multiply $f$ by $u|u|^{p-2}$ and integrate over $\Omega$ :

$$
\int_{\Omega} u_{t} u|u|^{p-2}-(\mathbf{A} u) u|u|^{p-2}+\mathbf{F}(u) u|u|^{p-2} \mathrm{~d} x=\int_{\Omega} f u|u|^{p-2} \mathrm{~d} x .
$$

Because $\frac{\partial}{\partial t}|u|^{p}=p u_{t} u|u|^{p-2}$, we have

$$
\int_{\Omega} u_{t} u|u|^{p-2} \mathrm{~d} x=\frac{1}{p} \frac{\mathrm{d}}{\mathrm{d} t}\|u(t, \cdot)\|_{L^{p}(\Omega)}^{p} .
$$

We use (H-A3) to reduce $-\int(\mathbf{A} u) u|u|^{p-2} \mathrm{~d} x$ to $C \int_{\Omega}|u|^{p} \mathrm{~d} x$, and we use (H-F4) to reduce $\int \mathbf{F}(u) u|u|^{p-2} \mathrm{~d} x$ to 0 . Thus, from equation (6.4) we deduce the inequality

$$
\frac{1}{p} \frac{\mathrm{d}}{\mathrm{d} t}\|u\|_{L^{p}(\Omega)}^{p}+C \int_{\Omega}|u|^{p} \mathrm{~d} x \leq \int_{\Omega} f u|u|^{p-2} \mathrm{~d} x .
$$

This holds for (almost) all $t \geq 0$. We integrate on $[0, \infty)$ to find that

$$
-\frac{1}{p}\|g\|_{L^{p}(\Omega)}^{p}+C\|u\|_{L^{p}((0, \infty) \times \Omega)}^{p} \leq \int_{[0, \infty) \times \Omega} f u|u|^{p-2} \mathrm{~d} t \mathrm{~d} x .
$$

Recall that $\|f\|_{p} \leq R$. With use of the Hölder inequality, we deduce from (6.6) that

$$
C\|u\|_{L^{p}((0, \infty) \times \Omega)}^{p} \leq \frac{1}{p}\|g\|_{L^{p}(\Omega)}^{p}+R\|u\|_{L^{p}((0, \infty) \times \Omega)}^{p-1} .
$$

By the embedding of $W^{1, p}\left((0, \infty), L^{p}(\Omega)\right)$ into $C^{0}\left([0, \infty), L^{p}(\Omega)\right)$, there is a constant $k>0$ depending only on $p$ and $\Omega$ such that $\|\cdot\|_{L^{p}(\Omega)} \leq k\|\cdot\|_{\mathcal{W}(0)}$ on $\mathcal{W}(0)$, and we have assumed that $\|g\|_{\mathcal{W}(0)} \leq R$. Therefore

$$
C\|u\|_{L^{p}((0, \infty) \times \Omega)} \leq \frac{(k R)^{p}}{\|u\|_{L^{p}((0, \infty) \times \Omega)}^{p-1}}+R .
$$


This shows that the choice $C_{p}=\max \left(1, C^{-1}\left(k^{p} R^{p}+R\right)\right)$ works.

For later use in verifying hypotheses of Theorem 5.3, we pause for the following corollary to the proof of Lemma 6.7 .

COROllary 6.8. In the situation of Lemma 6.7, if $u_{t}-\mathbf{A} u+\mathbf{F}(u)=0$ and $u(0)=0$, then $u=0$. Similarly, suppose that $[0, \infty)$ is replaced by $\mathbb{R}$. If $u \in \mathcal{W}_{\mathbb{R}}$ is such that $u_{t}-\mathbf{A} u+\mathbf{F}(u)=0$ on $\mathbb{R}$, then $u=0$.

Proof. For the first assertion, we return to the proof of Lemma 6.7, but with $R=0$ and $u(0)=0$. No changes are necessary up to estimate (6.6), which becomes $0+C\|u\|^{p} \leq 0$ as claimed. For the second assertion, we integrate (6.5) on $\mathbb{R}$ instead of on $[0, \infty)$. Now the first term in (6.6) is replaced by zero, and so $u=0$ once again.

6.2. Bounds in $\mathbf{L}^{\infty}$. Before proceeding to obtain bounds in $L^{\infty}$, we pause to explain the simple idea that may be hidden by some technicalities. Suppose for the moment that $u$ is smooth and that $A(t)=\Delta$. The function $|u|$ achieves its maximum value at some point $\left(t_{0}, x_{0}\right) \in[0, \infty) \times \Omega$, because $|u(t, x)| \rightarrow 0$ uniformly in $x$ as $t \rightarrow \infty$ and because $u$ vanishes on $[0, \infty) \times \partial \Omega$. If $t_{0}=0$, then we can easily bound $\|u\|_{\infty}$ by a constant that depends only on $\|u(0)\|_{\mathcal{W}(0)}$. Otherwise, $\left(t_{0}, x_{0}\right)$ lies in the open set $(0, \infty) \times \Omega$. Supposing that $u\left(t_{0}, x_{0}\right)>0$, it follows that $\frac{\partial u}{\partial t}\left(t_{0}, x_{0}\right)=0$ and that $-\Delta u\left(t_{0}, x_{0}\right) \geq 0$. Therefore, $\mathbf{F}(u) \leq f:=u_{t}-\Delta u+\mathbf{F}(u)$ at the point $\left(t_{0}, x_{0}\right)$. Since $u\left(t_{0}, x_{0}\right)>0$, we know from Hypothesis $(\mathrm{H}-\mathrm{F} 4)$ that $\mathbf{F}(u) \geq 0$ at $\left(t_{0}, x_{0}\right)$. With Hypothesis (H-F5), this gives an implicit bound for $\|u\|_{\infty}$ in terms of $\|f\|_{\infty}$. The case that $u\left(t_{0}, x_{0}\right)<0$ leads to a similar analysis.

The main problem at hand is to remove the assumption that $u$ is smooth. To begin, we recall some standard properties of smoothing by convolution. We bring in the standard mollifier $\eta: \mathbb{R} \times \mathbb{R}^{d} \rightarrow \mathbb{R}$ :

$$
\eta(y):= \begin{cases}\gamma \exp \left(\left(|y|^{2}-1\right)^{-1}\right) & \text { if }|y|^{2}<1 \\ 0 & \text { otherwise }\end{cases}
$$

where $\gamma>0$ is chosen so that $\int \eta=1$. It is a standard result that $\eta \in C_{0}^{\infty}\left(\mathbb{R} \times \mathbb{R}^{d}\right)$. As usual we take $\eta_{\epsilon}(y)=\epsilon^{-(d+1)} \eta(y / \epsilon)$ for each $\epsilon>0$. For any locally integrable function $u:[0, \infty) \times \Omega \rightarrow \mathbb{R}$, the $\eta_{\epsilon}$-mollification of $u$ is $u^{(\epsilon)}=\eta_{\epsilon} * u:(\epsilon, \infty) \times \Omega_{\epsilon} \rightarrow$ $\mathbb{R}$. Here, $\left(\eta_{\epsilon} * u\right)(y):=\int_{B(y ; \epsilon)} \eta_{\epsilon}(y-z) u(z) \mathrm{d} z$ and $\Omega_{\epsilon}:=\{x \in \Omega \mid \operatorname{dist}(x, \partial \Omega)>\epsilon\}$. Also, we are using $B(y ; \epsilon)$ for $\{z|| y-z \mid<\epsilon\}$. We shall make use of the following standard results, which can be found in $[\mathbf{1 0}] .^{5}$

LEMMA 6.9. With the above definitions come the following properties.

(1) $u^{(\epsilon)} \in C^{\infty}\left((\epsilon, \infty) \times \Omega_{\epsilon}\right)$.

(2) If $\alpha$ is any multi-index, then $D^{\alpha} u^{(\epsilon)}=\left(D^{\alpha} \eta_{\epsilon}\right) * u$.

(3) If $u$ is continuous on $(0, \infty) \times \Omega$, then $u^{(\epsilon)} \rightarrow u$ uniformly as $\epsilon \rightarrow 0^{+}$, on each compact subset of $(0, \infty) \times \Omega$.

Lemma 6.10. Let $A$ satisfy (H-A1)-(H-A4) and let F satisfy (H-F1)-(H-F5). Let $R>0$. There exists a constant $C_{\infty}=C_{\infty}(R)>0$ such that $\|u\|_{\infty} \leq C$ whenever $u \in \mathcal{W}$ with $\left\|u_{t}-\mathbf{A} u+\mathbf{F}(u)\right\|_{\infty} \leq R$ and $\|u(0)\|_{\mathcal{W}(0)} \leq R$.

\footnotetext{
${ }^{5}$ Assertion (1) is Theorem 6, part (i) of Appendix $\mathrm{C}$ in [10], and assertion (2) is derived as the proof of the same. Assertion (3) is Theorem 6, part (iii) of Appendix C in [10].
} 
Proof. The supremum of $|u(t, x)|$ is attained as a maximum at one or more points $(\tilde{t}, \tilde{x})$ in $[0, \infty) \times \Omega$, since $u$ is continuous, $u(t, x) \rightarrow 0$ as $t \rightarrow \infty$, and $u=0$ on $[0, \infty) \times \partial \Omega$. Let $\tilde{t}$ be the least possible value. There is a constant $k>0$ depending only on $p$ and $\Omega$ such that $\|\cdot\|_{L^{\infty}(\Omega)} \leq k\|\cdot\|_{\mathcal{W}(0)}$ on $\mathcal{W}(0)$, and we have assumed that $\|u(0)\|_{\mathcal{W}(0)} \leq R$. Hence, if $\tilde{t}=0$ the choice $C_{\infty}=k R$ works.

Now assume that $\tilde{t}>0$. We suppose that $u(\tilde{t}, \tilde{x})>0$; the analysis of the remaining case that $u(\tilde{t}, \tilde{x})<0$ is similar. Let $K=u^{-1}\left(\left\{\|u\|_{\infty}\right\}\right)$, which is a compact subset of $(0, \infty) \times \Omega$. Let $V$ be a bounded open set such that $K \subset V \subset$ $\bar{V} \subset(0, \infty) \times \Omega$.

Now consider a sequence $\left\{\epsilon_{n}\right\}$ of positive numbers that converges monotonically to zero. In doing so, choose $\epsilon_{1}$ sufficiently small that $\bar{V} \subset\left(\epsilon_{1}, \infty\right) \times \Omega_{\epsilon_{1}}$. For each $n \in \mathbb{N}$, let $u_{n}=\eta_{\epsilon_{n}} * u$, and choose a point $y_{n}=\left(t_{n}, x_{n}\right) \in \bar{V}$ where $\left.u_{n}\right|_{\bar{V}}$ assumes its maximum value. Without loss of generality, the sequence $\left\{y_{n}\right\}$ converges to a point $y_{0} \in \bar{V}$. By the uniform convergence of $\left\{u_{n}\right\}$ to $u$ on $\bar{V}$, and because $K \subset V$, it follows that

$$
u\left(y_{0}\right)=\lim _{n \rightarrow \infty} u_{n}\left(y_{n}\right)=\lim _{n \rightarrow \infty}\left\|u_{n}\right\|_{\infty}=\|u\|_{\infty}
$$

Now consider the sequence $\left\{\phi_{n}\right\}$, where we set $\phi_{n}(y):=\eta_{\epsilon_{n}}\left(y_{n}-y\right)$. Since $y_{n}$ is always in $\bar{V}$, we find that $\phi_{n}$ is always compactly supported in $(0, \infty) \times \Omega$. Since each $\phi_{n}$ is also smooth, we let $f:=u_{t}-\mathbf{A} u+\mathbf{F}(u)$ act on each $\phi_{n}$ as a distribution. Therefore,

$$
\int_{[0, \infty) \times \Omega} u_{t} \phi_{n}-(\mathbf{A} u) \phi_{n}+\mathbf{F}(u) \phi_{n} \mathrm{~d} y=\int_{[0, \infty) \times \Omega} f \phi_{n} \mathrm{~d} y .
$$

For each of the four terms that appear in the integrals in equation (6.8), we analyze what happens for large $n$. First, for $n$ sufficiently large that $y_{n}$ is in $V\left(\left\{y_{n}\right\}\right.$ converges to $\left.y_{0} \in K \subset V\right)$, the smooth function $u_{n}$ has a local maximum at $y_{n}$. Therefore, $\frac{\partial u_{n}}{\partial t}\left(y_{n}\right)=0$ for large $n$. Using integration by parts and property (2) in Lemma 6.9, we find that for all such $n$,

$$
\int_{[0, \infty) \times \Omega} u_{t} \phi_{n} \mathrm{~d} y=\left(\frac{\partial \eta_{\epsilon_{n}}}{\partial t} * u\right)\left(y_{n}\right)=\frac{\partial u_{n}}{\partial t}\left(y_{n}\right)=0 .
$$

Second, let $A_{n}$ denote the operator resulting from holding the coefficients $a^{i j}, b^{i}$, and $c$ in $A$ constant, by evaluation at $y_{n}=\left(t_{n}, x_{n}\right)$. It follows from the ellipticity of $A_{n}$ and the nonnegativity of $c$ that $-A_{n} u_{n}\left(y_{n}\right) \geq 0$. If we replace $A$ by $A_{n}$ in (6.8) and integrate by parts (which does not affect the constant coefficients of $A_{n}$ ) we find that

$$
-\int_{[0, \infty) \times \Omega}\left(\mathbf{A}_{n} u\right) \phi_{n} \mathrm{~d} y=\left(\mathbf{A}_{n} u_{n}\right)\left(y_{n}\right) \geq 0 .
$$


On the other hand, note that by the Hölder continuity (H-A4) of $a^{i j}$,

$$
\begin{gathered}
\left|\int_{[0, \infty) \times \Omega}\left(a^{i j}\left(y_{n}\right)-a^{i j}(y)\right) \frac{\partial^{2} u}{\partial x_{i} \partial x_{j}} \phi_{n} \mathrm{~d} y\right| \\
\leq\left(\int_{B\left(y_{n} ; \epsilon_{n}\right)}\left|\frac{\partial^{2} u}{\partial x_{i} \partial x_{j}}\right|^{p} \mathrm{~d} y\right)^{1 / p} . \\
\cdot C\left\{\int_{B\left(y_{n} ; \epsilon_{n}\right)}\left(\left|y_{n}-y\right|^{k} \epsilon_{n}^{-(d+1)} \eta\left(\left(y_{n}-y\right) / \epsilon_{n}\right)\right)^{p^{\prime}} \mathrm{d} y\right\}^{1 / p^{\prime}},
\end{gathered}
$$

where $k=(d+1) / p$ is the Hölder exponent and the constant $C$ is valid throughout $V$. The first factor on the right side of (6.10) converges to zero as $n \rightarrow \infty$, since $\frac{\partial^{2} u}{\partial x_{i} \partial x_{j}}$ is in $L^{p}$ and the volume of $B\left(y_{n} ; \epsilon_{n}\right)$ goes to zero. To show that the second factor is bounded, observe that the change of variables $z=\left(y_{n}-y\right) / \epsilon_{n}$ results in

$$
\begin{aligned}
\epsilon_{n}^{-p^{\prime}(d+1)} \int_{B\left(y_{n} ; \epsilon_{n}\right)} & \left|y_{n}-y\right|^{k p^{\prime}} \eta\left(\frac{y_{n}-y}{\epsilon_{n}}\right)^{p^{\prime}} \mathrm{d} y \\
& =\epsilon_{n}^{-p^{\prime}(d+1)} \int_{B(0 ; 1)}\left|\epsilon_{n} z\right|^{k p^{\prime}}(\eta(z))^{p^{\prime}} \epsilon_{n}^{d+1} \mathrm{~d} z \\
& =\int_{B(0 ; 1)}|z|^{k p^{\prime}}(\eta(z))^{p^{\prime}} \mathrm{d} z
\end{aligned}
$$

since the exponent $k p^{\prime}-(d+1)\left(p^{\prime}-1\right)$ of $\epsilon_{n}$ is zero by choice of $k$. Since this is a constant, we conclude that (6.10) tends to zero as $n \rightarrow \infty$.

A similar analysis applies to the first order terms (using Hölder continuity of $\tilde{b}^{i}$ ) and the zeroth order term (using only the continuity of $c$ ). With (6.9) it follows that

$$
\liminf _{n \rightarrow \infty} \int_{[0, \infty) \times \Omega}-(\mathbf{A} u) \phi_{n} \mathrm{~d} t \mathrm{~d} x \geq 0 .
$$

Third, since $\mathbf{F}(u)$ is continuous, $\left\{\eta_{\epsilon_{n}} * \mathbf{F}(u)\right\}$ converges uniformly on compact sets to $\mathbf{F}(u)$. Therefore,

$$
\left\{\int \mathbf{F}(u) \phi_{n}\right\}=\left\{\left(\eta_{\epsilon_{n}} * \mathbf{F}(u)\right)\left(t_{n}, x_{n}\right)\right\}
$$

converges to

$$
F\left(t_{0}, x_{0}, u\left(t_{0}, x_{0}\right)\right)=F\left(t_{0}, x_{0},\|u\|_{\infty}\right) \text { as } n \rightarrow \infty .
$$

Fourth and finally, $\int f \phi_{n} \leq\|f\|_{\infty} \leq R$ for all $n$.

All together, we let $n \rightarrow \infty$ in (6.8) to find that

$$
F\left(t_{0}, x_{0},\|u\|_{\infty}\right) \leq R \text {. }
$$

With hypothesis (H-F5), this gives an implicit a priori bound for $\|u\|_{\infty}$.

REMARK 6.11. We point out that hypothesis (H-F5) could be weakened to the following form:

$$
\rho(F):=\lim _{|z| \rightarrow \infty} \inf \{|F(t, x, z)| \mid t \geq 0, x \in \Omega\}>0 .
$$


That is, $F$ is bounded uniformly away from zero for large enough $z$. In this case, one should take $R<\rho(F)$ in the above lemma.

6.3. Bounds in $\mathcal{W}$. We will now use the a priori bounds in $L^{p}$ and $L^{\infty}$ to find bounds in $\mathcal{W}$, as required by condition (4) of Theorem 5.3.

Lemma 6.12. Let $A$ satisfy (H-A1)-(H-A4) and let F satisfy (H-F1)-(H-F5). Let $R>0$. There exists a constant $C=C(R)>0$ such that $\|u\|_{\mathcal{W}} \leq C$ whenever $u \in \mathcal{W}$ with $\left\|u_{t}-\mathbf{A} u+\mathbf{F}(u)\right\|_{p} \leq R,\|u(0)\|_{\mathcal{W}(0)} \leq R$, and $\left\|u_{t}-\mathbf{A} u+\mathbf{F}(u)\right\|_{\infty} \leq$ $R$.

Proof. Once again, put $f=u_{t}-\mathbf{A} u+\mathbf{F}(u)$ and $g=u(0)$. We are assuming that $u \in \mathcal{W}$ with $\|f\|_{p} \leq R,\|f\|_{\infty} \leq R$, and $\|u(0)\|_{\mathcal{W}(0)} \leq R$. Accordingly, let the bounds $C_{p}$ on $\|u\|_{p}$ and $C_{\infty}$ on $\|u\|_{\infty}$ be given by Lemmas 6.7 and 6.10 respectively.

By (H-A2) (see also Remark 3.3), $\left(\frac{\mathrm{d}}{\mathrm{d} t}-\mathbf{A}, E_{0}\right)$ is an isomorphism of $\mathcal{W}$ onto $\mathcal{X} \times \mathcal{W}(0)$. It follows that

$$
\begin{aligned}
\|u\|_{\mathcal{W}} & \leq k\left(\|f\|_{\mathcal{X}}+\|\mathbf{F}(u)\|_{\mathcal{X}}+\|g\|_{\mathcal{W}(0)}\right) \\
& \leq k\left(2 R+\|\mathbf{F}(u)\|_{\mathcal{X}}\right)
\end{aligned}
$$

where $k=\left\|\left(\frac{\mathrm{d}}{\mathrm{d} t}-\mathbf{A}, E_{0}\right)^{-1}\right\|$. According to (H-F2), let $M>0$ be a bound for $|F(t, x, z)|$ on $[0, \infty) \times \Omega \times\left[-C_{\infty}, C_{\infty}\right]$. We then have

$$
|\mathbf{F}(u)(t, x)| \leq \int_{0}^{1}\left|D_{z}(F(t, x, s u(t, x))) u(t, x)\right| \mathrm{d} s \leq M|u(t, x)| .
$$

From this, it follows that $\|\mathbf{F}(u)\|_{p} \leq M C_{p}$. With (6.12), it follows that $\|u\|_{\mathcal{W}} \leq$ $C:=k\left(2 R+M C_{p}\right)$.

6.4. Conclusion. We now illustrate the use of Theorem 5.3.

TheOREM 6.13. Suppose A satisfies (H-A1)-(H-A4) and that F satisfies (H-F1)(H-F5). For all $f \in L^{p}((0, \infty) \times \Omega) \cap L^{\infty}((0, \infty) \times \Omega)$ and all $g \in \mathcal{W}(0)$, there is a (strong) solution $u \in \mathcal{W} \subset W_{p}^{1,2}((0, \infty) \times \Omega)$ to the problem

$$
\begin{aligned}
u_{t}-A(t) u+F(t, x, u) & =f(t, x) \quad \text { in }[0, \infty) \times \Omega, \\
u(0, \cdot) & =g(x) \quad \text { in } \Omega, \\
u & =0 \quad \text { on }[0, \infty) \times \partial \Omega .
\end{aligned}
$$

Proof. Condition 1 in Theorem 5.3 holds by (H-A2). Condition 2 follows from Corollary 6.8.

For the third condition, let $B \in \omega(A)$ and $G \in \omega(F)$. By Lemma 4.7, $B$ satisfies (H-A1) with $A$ replaced by $B$ and $[0, \infty)$ replaced by $\mathbb{R}$. The uniformity of (H-A3) with respect to $t$ implies that this is also inherited by $B$. Similarly, by Lemma 4.5 the function $G$ satisfies (H-F1)- $(\mathrm{H}-\mathrm{F} 3)$ with $F$ replaced by $G$ and with $[0, \infty)$ replaced by $\mathbb{R}$. The uniformity of $(\mathrm{H}-\mathrm{F} 4)$ with respect to $t$ shows that this is also inherited by $G$. Therefore, it follows from Corollary 6.8 that the only $u \in \mathcal{W}_{\mathbb{R}}$ such that $u_{t}-\mathbf{B} u+\mathbf{G}(u)=0$ is $u=0$. This verifies the third numbered condition in Theorem 5.3.

Finally, for all $f \in L^{p}((0, \infty) \times \Omega) \cap L^{\infty}((0, \infty) \times \Omega)$ and all $g \in \mathcal{W}(0)$, Lemma 6.12 shows that $f$ and $g$ satisfy the fourth condition of Theorem 5.3. Indeed, in Lemma 6.12 use $R=\max \left\{\|f\|_{p},\|f\|_{\infty},\|g\|_{\mathcal{W}(0)}\right\}$. The resulting bound $C$ 
becomes the new $R$ for Definition 5.1, in which one can use the (norm decreasing) linear path $(s f, s g)$. This completes the proof.

\section{References}

[1] R. A. Adams and J. J. F. Fournier, Sobolev spaces, 2nd ed., Pure and Applied Mathematics, vol. 140, Elsevier Science Ltd., 2003.

[2] H. Amann, Quasilinear parabolic problems via maximal regularity, Adv. Differential Equations 10 (2005), no. 10, 1081-1110.

[3] P. Baras and R. Kersner, Local and global solvability of a class of semilinear parabolic equations, J. Differential Equations 68 (1987), no. 2, 238-252.

[4] A. N. Carvalho and J. W. Cholewa, Continuation and asymptotics of solutions to semilinear parabolic equations with critical nonlinearities, J. Math. Anal. Appl. 310 (2005), no. 2, 557578.

[5] A. Constantin, J. Escher, and Z. Yin, Global solutions for quasilinear parabolic systems, J. Differential Equations 197 (2004), no. 1, 73-84.

[6] K. Deimling, Nonlinear functional analysis, Springer-Verlag, Berlin, 1985.

[7] J. Diestel and J. J. Uhl, Jr., Vector measures, American Mathematical Society, Providence, R.I., 1977, With a foreword by B. J. Pettis, Mathematical Surveys, No. 15.

[8] N. Dunford and J. T. Schwartz, Linear operators. Part I, Wiley Classics Library, John Wiley \& Sons Inc., New York, 1988, General theory, With the assistance of William G. Bade and Robert G. Bartle, Reprint of the 1958 original, A Wiley-Interscience Publication.

[9] R. E. Edwards, Functional analysis. Theory and applications, Holt, Rinehart and Winston, New York, 1965.

[10] L. C. Evans, Partial differential equations, Graduate Studies in Mathematics, vol. 19, American Mathematical Society, Providence, RI, 1998.

[11] P.M Fitzpatrick, J. Pejsachowicz, and P.J. Rabier, The degree of proper $C^{2}$ Fredholm mappings. I, J. Reine Angew. Math. 427 (1992), 1-33.

[12] H. Fujita, On the blowing up of solutions of the Cauchy problem for $u_{t}=\Delta u+u^{1+\alpha}$, J. Fac. Sci. Univ. Tokyo Sect. I 13 (1966), 109-124 (1966).

[13] Y. Gu and W. Sun, Existence and nonexistence of global solutions for semilinear heat equation on unbounded domain, J. Partial Differential Equations 17 (2004), no. 4, 351-368.

[14] D. Henry, Geometric theory of semilinear parabolic equations, Lecture Notes in Mathematics, vol. 840, Springer-Verlag, Berlin, 1981.

[15] M. Kirane and N.-E. Tatar, Global existence and stability of some semilinear problems, Arch. Math. (Brno) 36 (2000), no. 1, 33-44.

[16] T. A. Kwembe, A remark on the existence and uniqueness of solutions of a semilinear parabolic equation, Nonlinear Anal. 50 (2002), no. 3, Ser. A: Theory Methods, 425-432.

[17] Gary M. Lieberman, Second order parabolic differential equations, World Scientific Publishing Co. Inc., River Edge, NJ, 1996.

[18] N. G. Lloyd, Degree theory, Cambridge University Press, Cambridge, 1978, Cambridge Tracts in Mathematics, No. 73.

[19] A. Lunardi, Analytic semigroups and optimal regularity in parabolic problems, Progress in Nonlinear Differential Equations and their Applications, 16, Birkhäuser Verlag, Basel, 1995.

[20] L. Maatoug and L. Riahi, Global existence of positive solutions for semilinear parabolic equations in a half-space, Differential Integral Equations 17 (2004), no. 11-12, 1273-1292.

[21] M. L. Marinov and V. S. Georgiev, Global existence of solution to the semilinear heat equation, C. R. Acad. Bulgare Sci. 42 (1989), no. 6, 21-23.

[22] J. R. Morris, Nonlinear ordinary and partial differential equations on unbounded domains, Ph.D. thesis, University of Pittsburgh, 2005, available at http://etd.library.pitt.edu/ETD/available/etd-03022005-112519/.

[23] A. Pazy, Semigroups of linear operators and applications to partial differential equations, Applied Mathematical Sciences, vol. 44, Springer-Verlag, New York, 1983.

[24] J. Pejsachowicz and P. J. Rabier, Degree theory for $C^{1}$ Fredholm mappings of index 0, J. Anal. Math. 76 (1998), 289-319.

[25] R. G. Pinsky, Global existence/nonexistence of sign-changing solutions to $u_{t}=\Delta u+|u|^{p}$ in $\mathbf{R}^{d}$, Bull. London Math. Soc. 37 (2005), no. 3, 417-426. 
[26] P. Poláčik and E. Yanagida, On bounded and unbounded global solutions of a supercritical semilinear heat equation, Math. Ann. 327 (2003), no. 4, 745-771.

[27] P. J. Rabier, An isomorphism theorem for linear evolution problems on the line, J. Dynam. Differential Equations 15 (2003), no. 4, 779-806.

[28] P. J. Rabier, The Robbin-Salamon index theorem in Banach spaces with UMD, Dyn. Partial Differ. Equ. 1 (2004), no. 3, 303-337.

[29] P.J. Rabier, Ascoli's theorem for functions vanishing at infinity and selected applications, J. Math. Anal. Appl. 290 (2004), no. 1, 171-189.

[30] P.J. Rabier and C. A. Stuart, Boundary value problems for first order systems on the halfline, Topol. Methods Nonlinear Anal. 25 (2005), no. 1, 101-133.

[31] — A Sobolev space approach to boundary value problems on the half-line, Commun. Contemp. Math. 7 (2005), no. 1, 1-36.

[32] R. Racke, Global solutions to semilinear parabolic systems for small data, J. Differential Equations 76 (1988), no. 2, 312-338.

[33] R. Schnaubelt, Asymptotic behaviour of parabolic nonautonomous evolution equations, Functional analytic methods for evolution equations, Lecture Notes in Math., vol. 1855, pp. 401472 .

[34] - Asymptotically autonomous parabolic evolution equations, J. Evol. Equ. 1 (2001), no. $1,19-37$.

[35] J. Simon, Compact sets in the space $L^{p}(0, T ; B)$, Ann. Mat. Pura Appl. (4) 146 (1987), $65-96$.

[36] F. B. Weissler, Existence and nonexistence of global solutions for a semilinear heat equation, Israel J. Math. 38 (1981), no. 1-2, 29-40.

Department of Mathematics,University of Pittsburgh, Pittsburgh, PA 15260

Current address: Department of Mathematics, University of Alabama at Birmingham, 1300

University Boulevard, Birmingham, AL 35294-1170

E-mail address: morris@math.uab.edu 